\title{
A Context-Based Theory of Recency and Contiguity in Free Recall
}

\author{
Per B. Sederberg \\ Princeton University
}

\author{
Marc W. Howard \\ Syracuse University
}

\author{
Michael J. Kahana \\ University of Pennsylvania
}

\begin{abstract}
The authors present a new model of free recall on the basis of M. W. Howard and M. J. Kahana's (2002a) temporal context model and M. Usher and J. L. McClelland's (2001) leaky-accumulator decision model. In this model, contextual drift gives rise to both short-term and long-term recency effects, and contextual retrieval gives rise to short-term and long-term contiguity effects. Recall decisions are controlled by a race between competitive leaky accumulators. The model captures the dynamics of immediate, delayed, and continual distractor free recall, demonstrating that dissociations between short- and long-term recency can naturally arise from a model in which an internal contextual state is used as the sole cue for retrieval across time scales.
\end{abstract}

Keywords: recency, contiguity, free recall, context

The Law of Recency refers to the observation that memories of recent experiences come to mind more easily than memories from the distant past (T. Brown, 1824; Calkins, 1896). Given the ubiquitous nature of recency across time scales, memory tasks, and stimulus materials, it is not surprising that it has occupied center stage in theoretical analyses of memory over many decades (Crowder, 1976).

Whereas some students of memory have sought a common cause for the varied manifestations of recency (Crowder, 1982; Greene, 1986), others have posited distinct mechanisms for the recency effects observed at short and at long time scales (Atkinson \& Shiffrin, 1968). In support of a dual-store explanation of recency, Davelaar, GoshenGottstein, Ashkenazi, Haarmann, and Usher (2005) identified several striking differences between the recency effects observed in immediate free recall and continual distractor free recall. In immediate free recall, participants are asked to recall the list items, in any order, immediately following the last item presentation. In continual distractor free recall, participants are given a demanding distractor task following each list item. After the last period of distraction, they are asked to recall the items in any order (see Figure 1 for a graphical description of the free-recall tasks).

Davelaar et al. (2005) suggested that the existence of dissociations between short- and long-term recency calls into question

Per B. Sederberg, Department of Psychology and Center for the Study of Brain, Mind, and Behavior, Princeton University; Marc W. Howard, Department of Psychology, Syracuse University; Michael J. Kahana, Department of Psychology, University of Pennsylvania.

Support for the present study was provided by National Institutes of Health Grants MH055587 and MH061975 awarded to Michael J. Kahana, MH069938 awarded to Marc W. Howard, and MH072138 and MH080526 awarded to Per B. Sederberg. We thank Kenneth Norman, and Sean Polyn for helpful discussions and/or feedback on this manuscript and Chris Moore for his artistic representations of temporal context in Figure 2.

Correspondence concerning this article should be addressed to Per B. Sederberg, Green Hall, Princeton University, Princeton, NJ 08540. E-mail: persed@princeton.edu models that hypothesize a general forgetting process underlying recency phenomena observed at different time scales. One popular class of general forgetting models assumes that a time-varying internal context signal gives rise to recency in both short-term and long-term recall tasks (see Kahana, Howard, \& Polyn, 2008, for a review). Davelaar et al. (2005) instead proposed a model of free recall in which an activation-based short-term store (STS) produces recency in immediate free recall, and a time-varying context signal produces long-term recency via a weight-based long-term store (LTS) in continual distractor free recall.

We next review some of the major empirical phenomena observed in free recall and their relevance for single- and dual-store theories of episodic memory. We conclude the introduction with a discussion of dissociations between immediate and long-term recency effects.

\section{Single-Versus Dual-Store Accounts of Free Recall}

In delayed free recall, a filled distractor interval intervenes between the last item and the test, resulting in a dramatic reduction in the short-term recency effect observed in immediate free recall (Glanzer \& Cunitz, 1966; Postman \& Phillips, 1965). According to dual-store models (e.g., Atkinson \& Shiffrin, 1968; Davelaar et al., 2005; Raaijmakers \& Shiffrin, 1980), the recency effect in immediate free recall is due to a direct read-out from STS, which has a capacity of approximately two to five items. When no additional items can be retrieved from STS, recall continues with retrieval from LTS. Although easily accessible, items in STS are extremely sensitive to retroactive interference from incoming information. Consequently, the distractor interval clears items from STS, and retrieval in delayed free recall is based only on LTS.

Because in free recall the order of recall reflects the order in which items come to mind, recall transitions from one item to the next presumably reflect the organization of memory for the list items. To examine the effects of the temporal organization of the list on freerecall transitions, Kahana (1996) measured the conditional response 


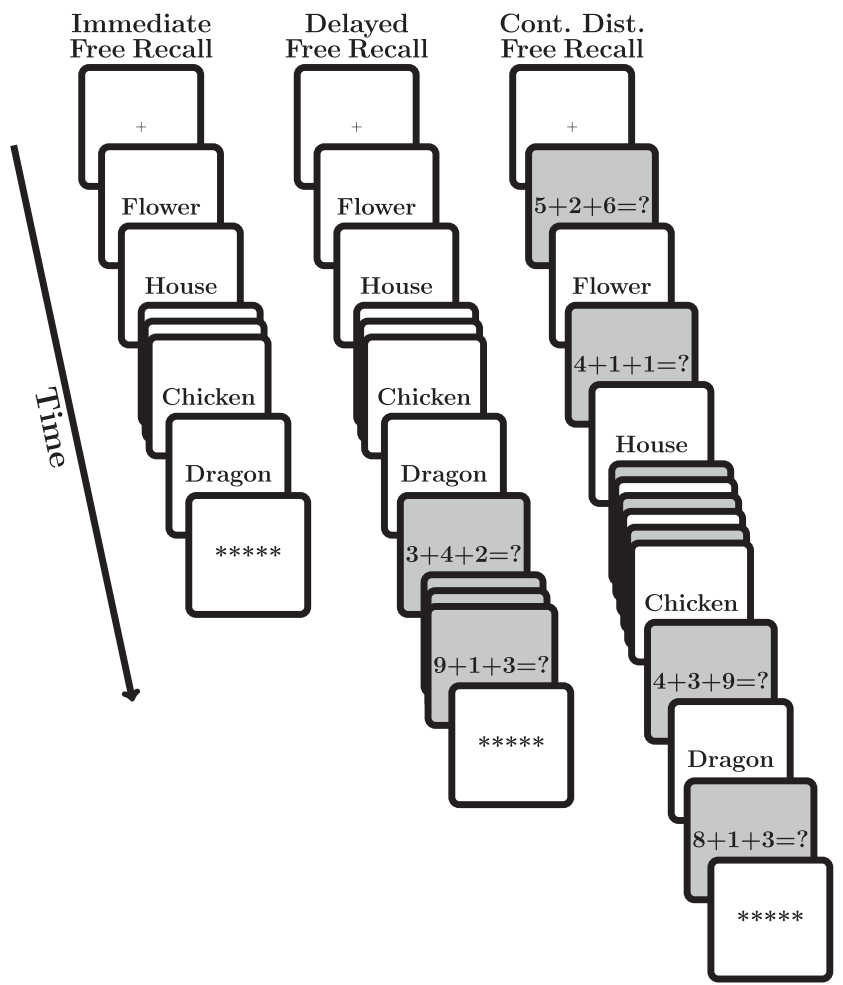

Figure 1. Graphical illustration of immediate, delayed, and continual distractor (Cont. Dist.) free recall. Each trial begins with a fixation cross. A row of asterisks signals participants to recall the items in any order.

probability as a function of lag (lag-CRP). Given that the participant has just recalled the item from serial position $i$, the lag-CRP indicates the probability that the next item recalled comes from serial position $i+$ lag. Lag-CRP analyses have shown that the contiguity effect, a tendency for participants to recall items from nearby in the list to the just-recalled item, and the asymmetry effect, a tendency for participants to recall items in the forward direction, are extremely robust properties of free recall (see Kahana et al., 2008, for a review).

In much the same way that dual-store models provide a natural account of recency effects in immediate and delayed recall, they also provide a natural explanation of the contiguity effect in immediate and delayed recall. The search of associative memory model (SAM; Kimball, Smith, \& Kahana, 2007; Mensink \& Raaijmakers, 1988, 1989; Raaijmakers \& Shiffrin, 1980, 1981; Sirotin, Kimball, \& Kahana, 2005), a detailed implementation of a dual-store model, postulates that connections are strengthened in long-term memory between items that are simultaneously active in STS. A contiguity effect arises because items from nearby positions in the list are likely to be coactive in STS. The interitem associations then provide a boost in the probability of transitioning to a nearby item during retrieval from LTS (Kahana, 1996). It should be noted that the co-occurance of items in STS does not by itself explain the ubiquitous asymmetry observed in the lag-CRP - this asymmetry must be added to SAM as an external constraint.

The ease with which the dual-store model addresses recency and contiguity effects in immediate and delayed free recall would give rise to an almost unquestioned adoption of dual-store models of memory were it not for the discovery of the long-term recency effect in continual distractor free recall (Bjork \& Whitten, 1974). In continual distractor free recall, there is a filled distractor interval not only between the last item and the recall test, as in delayed free recall, but also in the interval between the study of each list item. Buffer models of STS cannot account for recency in continual distractor free recall; if the end-of-list distractor in delayed free recall is sufficient to clear STS, then it should also be sufficient to clear STS in continual distractor free recall. Nonetheless, long-term recency effects are robustly observed in continual distractor free recall (Bjork \& Whitten, 1974; Glenberg, Bradley, Kraus, \& Renzaglia, 1983; Glenberg et al., 1980; Howard \& Kahana, 1999; Neath, 1993; Thapar \& Greene, 1993; Tzeng, 1973; Watkins, Neath, \& Sechler, 1989).

The discovery of long-term recency effects led Crowder (1982) and Greene (1986) to conclude that STS could not sufficiently explain recency effects in free recall. Whereas it is easy to postulate an additional recency mechanism in LTS to account for the long-term recency effect, ${ }^{1}$ similarities between immediate and long-term recency made it appealing to develop single-store models of memory that account for recency across time scales with a common mechanism.

Temporal distinctiveness models (G. D. A. Brown, Neath, \& Chater, 2007; Glenberg \& Swanson, 1986; Murdock, 1960; Nairne, Neath, Serra, \& Byun, 1997; Neath \& Crowder, 1990, 1996) assume that recall of an item depends not on its absolute recency, but on its relative recency to other list items. According to this view, continual distractor free recall results in larger recency than delayed free recall because the last item in the list is more distinctive in continual distractor free recall due to the fact that the delay in the interstimulus interval has placed the other items further into the past. Because the relative spacing of the list is similar in immediate and continual distractor free recall, temporal distinctiveness models can account for the existence of both immediate and long-term recency using the same mechanism. Temporal distinctiveness models often leave the mechanism by which a temporally varying signal is implemented as an abstract concept; time tags (Yntema \& Trask, 1963) or a randomly varying temporal context (Bower, 1972; Estes, 1955; Murdock, 1997) are two hypothesized candidates. The idea of a time-varying context representation has also been used to explain a wide range of interference phenomena (e.g., Kahana, in press; Mensink \& Raaijmakers, 1988).

Like the recency effect, the contiguity effect also persists when items are separated by an interval of distracting activity (Howard \& Kahana, 1999, see also Howard, Youker, \& Venkatadass, 2008). Following similar logic to that used in our discussion of the long-term recency effect, STS cannot simultaneously account for the contiguity effect observed in continual distractor free recall and the effect of a delay on the recency effect-if the interitem distractor is effective at clearing items from STS, then adjacent items would never be in STS at the same time in continual distractor free recall.

Howard and Kahana (2002a) proposed the temporal context model (TCM) to account for the pattern of results observed for recency and contiguity effects in free recall across presentation schedules. During study, items are associated with the current state of a gradually

${ }^{1}$ Atkinson and Shiffrin (1968) postulated that there was slow decay of information from LTS (see also Davelaar et al., 2005; Mensink \& Raaijmakers, 1988, 1989; Raaijmakers, 1993; Sirotin et al., 2005). 
changing representation of temporal context. The recency effect follows because items that were studied more recently are more similar to the time-of-test context. In TCM, the relative activation of the items in the list probabilistically determined which item was recalled. The contextual coding process along with the probabilistic choice rule enabled TCM to account for immediate and long-term recency using the same logic as distinctiveness models of long-term recency.

According to Howard and Kahana (2002a), temporal context is not independent of the items being presented. Rather than drifting randomly, as in stimulus fluctuation models (e.g., Estes, 1955; Mensink \& Raaijmakers, 1989), context changes from moment to moment in TCM because the items, themselves, drive the evolution of context. This property provides a natural account of contiguity effects-when an item is recovered at test, it reinstates the temporal context active when that item was studied. Because this context overlaps with the encoding context of the items' neighbors, a contiguity effect results. Because the retrieval rule is sensitive to the relative activation of the list items, TCM predicts a long-term contiguity effect for the same reason as it predicts the long-term recency effect.

Howard and Kahana (2002a) focused on modeling the contextual evolution and retrieval process, noting that TCM lacked much of the machinery needed to account for the major phenomena observed in free recall. To account for these phenomena, additional mechanisms would be needed. For instance, TCM lacked a stopping rule that would determine when recall terminates. It also lacked mechanisms to account for recall latency as well as rules to avoid repetition of already-recalled items.

The assertion, made by both TCM and the distinctiveness models, that short- and long-term recency could share a common source is not universally accepted. A number of experimental dissociations between short- and long-term recency have been observed. Davelaar et al. (2005) argued that these dissociations could not be readily accounted for by single-store models in general, and TCM in particular.

Here we show that dissociations between short- and long-term recency can be addressed within the framework of context-based recency models without postulating separate short-term and longterm memory stores. Building on Howard and Kahana's (2002a) model of temporal context, we present a model of free recall in which cuing with context produces both short-term and long-term recency effects. In our model, we used a retrieval rule based on the dynamic decision model of Usher and McClelland (2001). According to this model, each item accumulates strength on the basis of its activation and competes with the other items for recall via lateral inhibition. Using this retrieval rule, recall of an item depends on both its absolute level of activation and its level of activation relative to other list items. As in the classic stimulus sampling theory of Estes (1955, 1959), evolved context in our model can be seen as an activation-based memory representation. However, it is the retrieval of weight-based associations between context and items that determines retrieval of memories at both short and long time scales.

We start by reviewing the empirical dissociations between short- and long-term recency. We then present an overview of the TCM framework and TCM-A, a model of free recall in which competing accumulators are used to retrieve particular items given a contextual cue (Usher \& McClelland, 2001). A series of simulations demonstrate that TCM-A can address key dissociations between short- and long-term recency despite the fact that temporal context is the sole cue for recall in both short- and long-term recency experiments. More theoretical concerns about activationbased and weight-based memory are postponed until the General Discussion section.

\section{Dissociations Between Short- and Long-Term Recency}

Although there are many commonalities between short- and long-term recency in free recall (e.g., Greene, 1986), there are also a number of empirical dissociations between recency in these tasks. Two dissociations between recency in immediate and continual distractor free recall can be seen by examining the timing and order of participants' recalls. Although participants exhibit a similar tendency to begin recall at the end of the list in both tasks, they take longer to initiate recall in continual distractor free recall. Second, participants tend to recall several end-of-list items in succession in immediate free recall, whereas in continual distractor free recall, they tend to jump to earlier list items after recalling one or two items from the end of the list. This dissociation can be seen in the lag-CRP functions: In immediate free recall, the lag-CRP exhibits much stronger contiguity in early than in late output positions (Kahana, 1996). This is not the case in delayed or continual distractor free recall in which the contiguity effect is approximately constant across output positions (Howard \& Kahana, 1999). A third dissociation can be seen in studies of proactive interference. Whereas the recency effect in immediate free recall is relatively insensitive to proactive interference (Craik \& Birtwistle, 1971; Murdock, 1962), the recency effect in continual distractor free recall is reduced under conditions of high proactive interference (Davelaar et al., 2005). Finally, the long-term recency effect, but not the immediate recency effect, is disrupted in patients with anterograde amnesia (Carlesimo, Marfia, Loasses, \& Caltagirone, 1996). Below we discuss each of these dissociations.

\section{Dissociation: Time to First Recall}

A dissociation between immediate and continual distractor free recall can be seen in the time participants take to initiate recall. In immediate free recall, recall starts quickly with a burst of several items, typically from the end of the list (Kahana, 1996; Nilsson, Wright, \& Murdock, 1975). As recall proceeds, interresponse times increase with output position (Murdock \& Okada, 1970). Continual distractor free recall does not start with a quick burst of items, but starts slowly in a way that appears to require an effortful search. Analysis of response times from Howard and Kahana (1999) shows that the mean time to initiate recall was $1.04 \mathrm{~s}$ in immediate and $2.15 \mathrm{~s}$ in continual distractor free recall.

\section{Dissociation: Changes in the Contiguity Effect With Output Position}

Davelaar et al. (2005) noted that in immediate free recall, the contiguity effect measured for the first few recalls is much more pronounced than the contiguity effect observed at later output positions (Howard \& Kahana, 1999; Kahana, 1996; Kahana, Howard, Zaromb, \& Wingfield, 2002) but that this decrease in the contiguity effect with output position is not observed in delayed or continual distractor free recall (Howard \& Kahana, 1999; Kahana et al., 2002). 
According to buffer models, the change in the contiguity effect with output position occurs because participants begin recall by reporting all of the items available in STS (Davelaar et al., 2005; Kahana, 1996). The items in STS at the time of test tend to be from the end of the list. In immediate free recall, these adjacent items are recalled first. Later in recall, responses depend on retrieval from LTS, resulting in a reduced contiguity effect. According to two-store models, retrieval in continual distractor free recall takes place entirely from LTS, so there would be no reason for the contiguity effect to change with output position (Davelaar et al., 2005).

\section{Dissociation: Proactive Interference}

The short-term recency effect observed in immediate free recall is remarkably insensitive to proactive interference. This point was illustrated nicely by Murdock (1962), who showed that the magnitude of the recency effect in immediate free recall was the same for lists of 20,30, and 40 items (Murdock, 1974). One might have expected that recall would decrease with increasing list length due to the buildup of proactive interference. In fact, the robustness of short-term recency to proactive interference may be the single most compelling rationale for a short-term memory buffer-no matter how much information a participant has been exposed to over the course of their lives, information presented in the last couple of seconds remains accessible. ${ }^{2}$

Davelaar et al. (2005) presented an experiment in which they observed a reduction in the magnitude of the recency effect in continual distractor, but not in immediate free recall, due to proactive interference from semantically similar items on a previous list. It is reasonable to assume that presenting two lists of semantically similar items effectively increases the list length of the second list, and thus increases proactive interference, due to the semantic associations with the prior list items (e.g., Underwood, 1983).

\section{Dissociation: Anterograde Amnesia}

Carlesimo et al. (1996) reported a dissociation between shortand long-term recency, as measured by two tasks given to amnesic and control participants. They tested immediate recency by means of a standard immediate free-recall procedure with each of 10 list items presented for $5 \mathrm{~s}$. The test for long-term recency was quite different; they had participants solve lists of 10 anagrams, with $30 \mathrm{~s}$ for each anagram. Each anagram was preceded by a $10-\mathrm{s}$ distractor of backwards counting, and the last item was followed by $30 \mathrm{~s}$ of backwards counting. The behavioral results revealed no difference in recall for the last serial position in immediate free recall but showed a decrease in recall relative to controls at all positions in their variant of continual distractor free recall. These results were taken as support for dual-store models of free recall because the amnesics' presumably intact STS supports recall of recency items in immediate free recall, whereas their damaged LTS impairs recall of recency items in continual distractor.

Because the critical comparison of Carlesimo et al. (1996) is between two very different tasks, it is difficult to make strong inferences from their reported dissociation. Furthermore, their use of a very slow presentation rate would allow participants to engage in extensive rehearsal, thus further confounding the relationship between the items' nominal and functional serial positions (Brodie \& Murdock, 1977; G. D. A. Brown, Sala, Foster, \& Vousden,
2007; Tan \& Ward, 2000; Ward, Woodward, Stevens, \& Stinson, 2003). Nonetheless, the data are sufficiently interesting to warrant further theoretical investigation.

\section{Overview}

Howard and Kahana's (2002a) implementation of TCM lacked the mechanisms needed to account for a number of free-recall phenomenon, including the key short- versus long-term recency dissociations outlined above. We present a new model that combines the associative framework of TCM with a dynamical decision component. The new model, called TCM-A, replaces the old retrieval rule in TCM with a set of leaky, competitive accumulators representing the activations of the words in the list (Usher \& McClelland, 2001). As part of the general class of diffusion models (e.g., Ratcliff, 1978, 2006), the Usher and McClelland (2001) decision model has gained considerable currency in both psychology and neuroscience, providing realistic accounts of behavioral reaction times and neural activity during a wide variety of tasks (Bogacz, 2007; Bogacz, Usher, Zhang, \& McClelland, 2007; McMillen \& Holmes, 2006).

The novel property of TCM-A that enables it to account for the key dissociations between short- and long-term recency is that the accumulator retrieval rule is sensitive both to the relative and absolute activation of items in selecting a candidate item to recall. This, coupled with the gradual change of temporal context across output positions, ensures that the recency effect in immediate free recall extends over several output positions, whereas the recency effect in continual distractor free recall falls off more steeply, affecting primarily the last item.

The next sections provide an overview of the TCM framework and describe the implementation of TCM-A in detail, followed by the results of simulations compared with data from Howard and Kahana (1999). These simulations show that our elaborated version of TCM can account for the four dissociations described above. We conclude that although a separate working memory buffer may serve other useful cognitive functions (Jonides et al., 2007), it does not appear necessary to propose a mechanism in addition to a gradually changing state of temporal context to account for the recency and contiguity effects observed in free recall.

\section{TCM With Accumulating Retrieval Dynamics}

Howard and Kahana's (2002a) TCM describes how context is represented and how it evolves as a consequence of item encoding and retrieval. It also describes an associative architecture that links items to context and context to items. In this section, we describe TCM-A, a model of free recall in which the basic assumptions of TCM are implemented in a neural network that can flexibly store and retrieve episodic memories. A key component of TCM-A is a retrieval rule based on the Usher and McClelland (2001) competitive leaky-accumulator choice model. We first provide a nonmathematical walk-through of TCM-A's assumptions and their implications for modeling free-recall data. We then provide a more

\footnotetext{
${ }^{2}$ Whereas the recency effect in immediate free recall does not appear to be sensitive to proactive interference, proactive interference does affect immediate recognition memory for recently experienced items (e.g., Monsell, 1978; van Vugt, Sekuler, Wilson, \& Kahana, 2008).
} 
formal treatment of the model and its implementation. In our description of TCM-A below, we note aspects of the model that differ from the original TCM (Howard \& Kahana, 2002a; Howard, Kahana, \& Wingfield, 2006). ${ }^{3}$

\section{Overview of the TCM Framework}

At the heart of TCM is a representation of temporal context, which is composed of a set of feature values slowly drifting through a high-dimensional feature space. Whereas early theorists modeled temporal context as feature values that change over time due to random fluctuations in the input (Anderson \& Bower, 1972; Estes, 1955), in TCM, activation of item representations, either during presentation or retrieval, drives the evolution of context.

Prior to studying a list, TCM assumes that each list item is associated with a preexperimental contextual representation. This contextual representation would in general be an amalgam of many prior contexts in which the item has appeared, but for the sake of simplicity, we will assume that all prior contexts combine into a single aggregate representation. As a participant studies each list item, the item activates its preexperimental context via the stored item-to-context associative connections (see Figure 2). ${ }^{4}$ This preexperimental context representation is then added to the current state of temporal context, which must first decay to make room for the newly inserted item. This process, whereby each studied item activates its preexperimental context, which, in turn, is combined with the current context, causes the contextual mechanism to drift as each new item is encoded. Because previous states of context are not completely obliterated by incoming information, this induces a correlation in successive states of context.

To make this more concrete, suppose that a participant studied the list flower, house, chicken, and dragon. When flower is studied, it would activate flower-related information in memory (i.e., its preexperimental context). This information would enter the current context, indicated by the picture of a flower in the context layer of Figure 2. When house is studied, it would activate house-related contextual information, which would enter the current state of context, weakening the strength of the flower-related information in the evolving context representation. This would continue for each item presentation, so that by the time dragon is studied (as indicated by the double circle in the item layer of Figure 2), the current state of context would include the preexperimental contexts of flower, house, chicken, and dragon, with the dragon context being represented more strongly than the chicken context, which, in turn, would be represented more strongly than the house context, etc. (see the context layer at the bottom of Figure 2). By this mechanism, the contexts or thoughts evoked by each studied item push the internal context signal through a high-dimensional space.

As context evolves, the current state of context becomes associated with each studied item. This learning involves the creation of a new association between the state of context when that item was presented and its item representation. This enables subsequent states of context to cue for the item to the extent that it overlaps with this encoding state. In addition, an association is updated between the item representation and the contextual state, such that repeating an item will enable it to recover this newly learned state of context. As described in more detail below, these context-toitem and item-to-context associations are represented as networks of Hebbian connections in our neural network implementation.
At the beginning of the recall period, the time-of-test context serves as a retrieval cue, activating items via the context-to-item associations stored in memory. By this point, each studied item has been associated with an experimental list context in addition to a preexperimental context. Because recent list items (e.g., dragon) are most strongly represented in the time-of-test context, cuing with time-of-test context gives rise to greater activation of end-oflist items, which gives rise to the recency effect.

Although many items are activated to some extent by the contextual cue, only one item may be recalled at a time. The process by which an individual item is selected for recall involves a competition among a set of leaky accumulators that correspond to the items. As suggested in the top right of Figure 2, each item accumulates strength in the presence of noise on the basis of the degree to which it is activated by the cue context. Item accumulators inhibit other accumulators, until an accumulator corresponding to a not-yet-recalled item crosses a threshold. In this example, the items near the end of the list have the greatest activation, which is indicated by the size of each circle representing an item. Consequently, the accumulators corresponding to end-of-list items grow faster than those corresponding to earlier list items, and have an increased chance of crossing threshold first.

Once recalled, an item is re-presented to the item layer and used to update the current state of temporal context. The retrieval of an item also retrieves the state of temporal context that was present when it was first studied. Consequently, context is updated with a combination of the preexperimental and newly learned contextual representations of the just-recalled item. This new state of context now serves as the cue to recall further items.

The preexperimental contextual features for the just-recalled item provide a forward-asymmetric cue for nearby list items because they were present in context only after that item was presented during encoding. The newly learned contextual features that were encoded when that item was studied provide a bidirectional cue for nearby list items because those features were part of temporal context both before and after the item was presented. The combined forwardasymmetric and bidirectional cue for subsequent recalls gives rise to the characteristic asymmetric contiguity effect.

This recall process, in which the just-recalled item updates the current state of temporal context, which then provides a cue for the subsequent recall, continues until all the items are recalled or time runs out.

\section{Formal Description of TCM-A}

The evolution of context. The state of context at time Step $i, \mathbf{t}_{i}$, evolves due to the information that is currently being processed by the memory system. Mathematically, TCM-A models the context vector as evolving according to the equation:

$$
\mathbf{t}_{i}=\rho_{i} \mathbf{t}_{i-1}+\beta \mathbf{t}_{i}^{I N},
$$

where $\beta$ is a parameter that determines the rate of contextual drift during encoding, $\rho_{i}$ is a scaling parameter chosen at each time step

\footnotetext{
${ }^{3}$ Our TCM-A software may be freely obtained at http://memory .psych.upenn.edu/.

${ }^{4}$ Although we assume that these preexperimental contextual representations are independent across items, one could easily extend the model to allow for correlated contexts.
} 

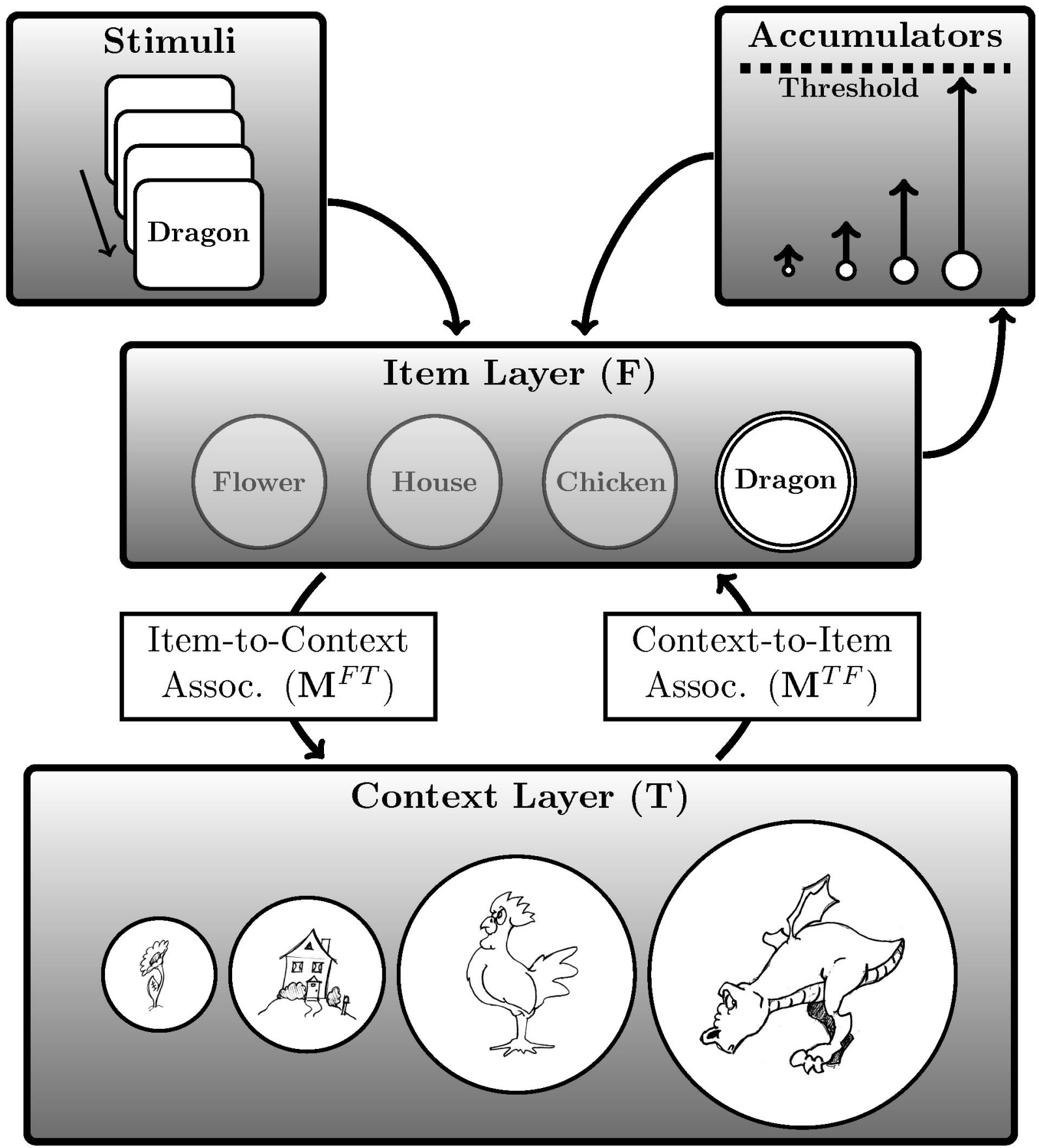

Figure 2. Temporal context model (TCM-A) with an accumulator-based retrieval rule. TCM-A is composed of three main layers: an item and a context layer, which are connected by associative matrices, and a layer of accumulators that provide a competitive retrieval mechanism. See the main text for a complete description of how these layers interact to store and retrieve episodic memories. Assoc. $=$ Association.

such that $\mathbf{t}_{i}$ is always of unit length, and $\mathbf{t}_{i}^{I N}$ is the input at time Step $i$ (throughout this article, we use boldface letters to denote vectors and matrices). $\mathbf{t}_{0}$ is the state of context prevailing when the first item, $\mathbf{f}_{1}$, is presented for study. In TCM-A, as in prior work on TCM, we assume that the vectors representing the studied items, denoted as $\mathbf{f}_{i}$, are orthonormal. As we explain below, the input pattern $\mathbf{t}_{1}^{I N}$ is the contextual information retrieved by item $\mathbf{f}_{1}$. Thus, after $\mathbf{f}_{1}$ has been studied, $\mathbf{t}_{1}=\rho_{1} \mathbf{t}_{0}+\beta \mathbf{t}_{1}^{I N}$. This is the context that prevails when the subsequent item, $\mathbf{f}_{2}$, is presented.

Hebbian associative memory. Matrices are used in TCM-A to represent item-to-context and context-to-item associations (see Figure 2). The context-to-item associations are stored in a matrix, 
$\mathbf{M}^{T F}$, which allows contextual states to cue items. The item-tocontext matrix, $\mathbf{M}^{F T}$, allows items to recover previous states of context. The input pattern $\mathbf{t}_{i}^{I N}$ that drives the evolution of context in Equation 1 is calculated from $\mathbf{M}^{F T}$ and the item $\mathbf{f}_{i}$ as:

$$
\mathbf{t}_{i}^{I N} \propto \mathbf{M}^{F T} \mathbf{f}_{i}
$$

where the proportionality symbol reflects the fact that $\mathbf{t}_{i}^{I N}$ is normalized to be of unit length before contributing to Equation 1 .

In TCM-A, we distinguish between preexperimental and experimentally learned item-to-context associations, which we denote as $\mathbf{M}_{\text {pre }}^{F T}$ and $\mathbf{M}_{\text {exp }}^{F T}$, respectively. These terms are weighted by a parameter $\gamma F T$ :

$$
\mathbf{M}^{F T}=\left(1-\gamma_{F T}\right) \mathbf{M}_{p r e}^{F T}+\gamma_{F T} \mathbf{M}_{e x p}^{F T}
$$

We also distinguish between preexperimental and experimentally learned context-to-item associations via $\mathbf{M}_{\text {pre }}^{T F}$ and $\mathbf{M}_{\text {exp }}^{T F}$, weighted similarly by a parameter $\gamma T F$ :

$$
\mathbf{M}^{T F}=\left(1-\gamma_{T F}\right) \mathbf{M}_{p r e}^{T F}+\gamma_{T F} \mathbf{M}_{e x p}^{T F} .
$$

This distinction between new (episodic) learning and longstanding (semantic) knowledge enables TCM-A to simulate performance in situations in which the mechanisms responsible for new associative learning have been damaged (Carlesimo et al., 1996). A similar assumption made in the eSAM and fSAM models proved crucial for simulating the interacting effects of preexperimental and new learning in categorized free recall (Sirotin et al., 2005) and in the Deese-Roediger-McDermott false-memory paradigm (Kimball et al., 2007).

For simplicity, we fix $\mathbf{M}_{\text {pre }}^{F T}$ and $\mathbf{M}_{\text {pre }}^{T F}$ as identity matrices that do not change during the encoding and recall of a simulated list. Clearly, these preexperimental weight matrices must change over time, but at a much slower time scale than the course of a single simulated list. The use of identity matrices for the preexperimental weights implies that there is no semantic similarity between items in the list. Although preexperimental interitem similarity does affect memory retrieval in free recall (Howard \& Kahana, 2002b), it is not necessary to build semantic similarity into the model to illustrate differences between short-term and long-term recency effects. Finally, the matrices representing experimental item-to-context associations, $\mathbf{M}_{\text {exp }}^{F T}$ and $\mathbf{M}_{\text {exp }}^{T F}$, are set to zero at the beginning of each simulated list and updated to learn the associations between items and context during the list presentation. ${ }^{5}$

Associations between context and items. TCM-A assumes that items become associated with context. We update a Hebbian outer product matrix, $\mathbf{M}_{\text {exp }}^{T F}$, that associates the prevailing context with the presented item's representation. This follows the equation:

$$
\Delta \mathbf{M}_{\text {exp }}^{T F}=\phi_{i} \mathbf{f}_{i} \mathbf{t}_{i-1}^{\top},
$$

where $T$ denotes the transpose operator (note that the transpose operator bears no relation to our use of $\mathbf{t}$ to denote the state of temporal context). ${ }^{6}$ As described in the Modeling primacy section, $\phi_{i}$ biases learning depending on the item's serial position in the list such that items from early serial positions are more strongly encoded. Similar assumptions have been used by computational models to describe primacy effects in serial recall (e.g., G. D. A Brown, Preece, \& Hulme, 2000; Burgess \& Hitch, 1999).

Associations between items and context. As with the contextto-item associations, we also increment the Hebbian outer product matrix, $\mathbf{M}_{\text {exp }}^{F T}$, that associates the active item to the state of context when that item was presented:

$$
\Delta \mathbf{M}_{\text {exp }}^{F T}=\mathbf{t}_{i-1} \mathbf{f}_{i}^{\top} .
$$

As a consequence of this learning in $\mathbf{M}_{\text {exp }}^{F T}$, recalling an item recovers the state of context prevailing just prior to its initial presentation.

While storing the newly learned associations between $\mathbf{t}_{0}$ and $\mathbf{f}_{1}$, we update the current state of temporal context according to Equation 1. Specifically, $\mathbf{t}_{1}=\rho_{1} \mathbf{t}_{0}+\beta \mathbf{t}_{1}^{I N}$. The first time an item is presented, only $\mathbf{M}_{\text {pre }}^{F T}$ contributes to the retrieved context because the newly learned association between that item and the experimental context has yet to be stored. For instance, $\mathbf{t}_{1}^{I N}$ will be given by the equation:

$$
\mathbf{t}_{1}^{I N}=\mathbf{M}_{p r e}^{F T} \mathbf{f}_{1} .
$$

Because $\mathbf{M}_{\text {pre }}^{F T}$ is an identity matrix, and the $\mathbf{f}_{i}$ s are orthonormal, this means that the input patterns caused by a list of unique items will also be orthonormal. Thus, the state of context following the presentation of $\mathbf{f}_{1}$ is simply a weighted sum of the prior state of context, $\mathbf{t}_{0}$, and the representation of $\mathbf{f}_{1}$. Following the presentation of $\mathbf{f}_{2}$, we would have $\mathbf{t}_{2}=\rho_{2} \mathbf{t}_{1}+\beta \mathbf{t}_{2}^{I N}=\rho_{2} \rho_{1} \mathbf{t}_{0}+\rho_{2} \beta \mathbf{t}_{1}^{I N}+\beta \mathbf{t}_{2}^{I N}$. Note that $\mathbf{t}_{2}^{I N}$ will have a larger weight than $\mathbf{t}_{1}^{I N}$ because $\rho_{i}<1$.

The order of encoding operations. In our simulations, when an item is presented during study, we first store the context-to-item association in $\mathbf{M}_{\text {exp }}^{T F}$ on the basis of the prevailing state of context when that item was presented (Equation 5). We next calculate $\mathbf{t}^{I N}$ according to Equation 2 but then update the item-to-context association in $\mathbf{M}_{\text {exp }}^{F T}$ (Equation 6) prior to using the $\mathbf{t}^{I N}$ calculated in the previous step to update context (Equation 1). That is, we do not allow the item-to-context weights to bind an item to the context that it has just retrieved. To some extent, this is simply a matter of convenience - there would be essentially no effect on the simulations we report here if this were not the case. ${ }^{7}$ One potential mechanism whereby items could avoid being bound to their own retrieved context is offered by the hypothesis that theta oscillations give rise to separate encoding and retrieval phases at region CA1 of the hippocampus (Hasselmo, Bodelon, \& Wyble, 2002). Siekmeier, Hasselmo, Howard, and Coyle (2007) showed how this mechanism could be used to modulate encoding

\footnotetext{
${ }^{5}$ Resetting the experimental association matrices between lists is a simplification to allow us to focus on intra- rather than interlist effects.

${ }^{6}$ In this implementation, we are assuming that the dimensionality of the $\mathbf{f}$ vectors is the same as the dimensionality of the $\mathbf{t}$ vectors. This is not a necessary feature of the model.

${ }^{7}$ If we did allow items to be bound to their own retrieved contexts, then we could recover the same simulation results with only changes to model parameters. Specifically, $\boldsymbol{t}_{i}{ }^{I N}$ would include an additional term of $\mathbf{t}_{i-1}$, which could be accounted for by decreasing $\beta$ relative to the model as implemented here. Although, the simulations we report would be identical, it may be that a meaningful difference between our implementation and one in which the items were bound to their own retrieved contexts would arise after many repeated item presentations.
} 
and retrieval of temporal context in much the same way we describe here.

Contextual states are used to retrieve item representations. According to dual-store models of free recall, retrieval begins by first reporting the items in STS, or those items whose activations exceed a threshold (Davelaar et al., 2005; Raaijmakers \& Shiffrin, 1981; Sirotin et al., 2005). It is this buffer mechanism that is responsible for the recency effect in immediate free recall and the recency dissociations between immediate and continual distractor free recall. Unlike these models, TCM-A assumes that recall begins by using the time-of-test context as a retrieval cue. The time-of-test context serves as a cue for recall of items via $\mathbf{M}^{T F}$.

Multiplying $\mathbf{M}^{T F}$ by the context vector, $\mathbf{t}_{\text {test }}$, retrieves a superposition of the items $\mathbf{f}_{i}$, each activated to the extent that the time-of-test context overlaps with both their encoding and preexperimental contexts stored in $\mathbf{M}^{T F}$ :

$$
\tilde{\mathbf{f}}=\mathbf{M}^{T F} \mathbf{t}_{\text {test }}=\left(1-\gamma_{T F}\right)\left(\mathbf{M}_{p r e}^{T F} \mathbf{t}_{\text {test }}\right)+\gamma_{T F}\left(\mathbf{M}_{\text {exp }}^{T F} \mathbf{t}_{\text {test }}\right),
$$

where the second line follows from the decomposition of $\mathbf{M}^{T F}$ (Equation 4). Multiplying Equation 8 from the left with $\mathbf{f}_{i}^{T}$, we find that the activation of each item $\tilde{\mathrm{f}}_{i}$ is:

$$
\tilde{\mathbf{f}}_{i}=\left(1-\gamma_{T F}\right)\left(\mathbf{t}_{i}^{I N} \cdot \mathbf{t}_{\text {test }}\right)+\gamma_{T F}\left(\mathbf{t}_{i-1} \cdot \mathbf{t}_{\text {test }}\right)
$$

because $\mathbf{f}_{i}^{\mathrm{T}} \mathbf{M}_{\text {pre }}^{T F}=\mathbf{t}_{i}^{I N}$ and $\mathbf{f}_{i}^{\mathrm{T}} \mathbf{M}_{\text {exp }}^{T F}=\mathbf{t}_{i-1}$. Thus, items that were originally seen in a study context $\left(\mathbf{t}_{i-1}\right)$ that are similar to the time-of-test context will be more strongly activated than those that were originally seen in a completely dissimilar context. Similarly, recently studied items will be more strongly activated because they will have greater representation ( $v i a \mathbf{t}_{i}^{I N}$ ) than early list items in the time-of-test context vector. This latter mechanism enables a recency effect in the absence of new learning at either $\mathbf{M}_{\text {exp }}^{T F}$ or $\mathbf{M}_{\text {exp }}^{F T}$. This property will turn out to be crucial in our account of anterograde amnesia.

We fixed $\gamma_{T F}$ at 0.8 (except for the amnesia simulations), which indicates that the item activations derived from the time-of-test context are based more on the newly learned experimental contextto-item associations than on the preexperimental associations. Although one could imagine that participants might dynamically bias retrieval to favor preexperimental/semantic or newly learned experimental/temporal associations, which would be equivalent to changing $\gamma_{T F}$ during the recall period, we do not consider such biasing effects in this article.

Accumulator-based retrieval mechanism. In TCM-A, we replaced the Luce choice retrieval rule (Howard and Kahana, 2002a) used in previous implementations of TCM with a set of competitive, leaky accumulators, as described in the Usher-McClelland model of perceptual decision making (Usher \& McClelland, 2001). The Usher-McClelland accumulator model has a number of desirable properties, including the ability to model competitive choice processes with realistic reaction times.

The item activations $\tilde{\mathbf{f}}$ derived from the current state of context (Equation 8) provide the input to the accumulators, scaled by the coefficient of variation $\left(C_{V}\right)$ of the item activations, defined as the standard deviation divided by the mean. The $C_{V}$ serves as a measure of dispersion in the input to the accumulators, scaling the input so the accumulators can optimally decide between the retrieval choices. To ensure that only relevant inputs are considered in the determination of the input dispersion, items whose activa- tions are below a minimum activation $\left(\tilde{\mathbf{f}}_{i}<\alpha_{\text {min }}\right.$ ) are not included in the calculation of the $C_{V}$. If the $C_{V}$ is greater than one, then the input is of high variance relative to its mean (i.e., the input is highly dispersed) and is scaled up to allow the accumulators to make a faster decision. Conversely, if the $C_{V}$ is less than 1 , then the input's variance is low relative to its mean (i.e., the input is less dispersed), and item activations are scaled down, giving the accumulators more time to differentiate between the items. In our simulations of immediate and delayed free recall, the $C_{V}$ factor is typically less than one and scales the inputs down at the start of recall, whereas in simulations of continual distractor free recall, the $C_{V}$ factor scales the inputs up at the start of recall. Finally, if the $C_{V}$ is undefined or zero, as is the case when the standard deviation or mean across item activations is zero, the $C_{V}$ remains unchanged from the previous recall or is set to one if there have been no previous responses.

At the start of recall, the accumulators $\mathbf{x}$ are initialized to zero. They then evolve over the course of a retrieval attempt according to the following equations:

$$
\begin{aligned}
& \mathbf{x}_{s}=(1-\tau \kappa-\tau \lambda \mathbf{L}) \mathbf{x}_{s-1}+\tau \boldsymbol{C}_{V} \tilde{\mathbf{f}}+\varepsilon \\
& \mathbf{x}_{\mathrm{s}} \rightarrow \max \left(\mathbf{x}_{s}, 0\right),
\end{aligned}
$$

where $\mathbf{x}_{s}$ is the new state of the vector of accumulators across all list items at retrieval Time Step $s, C_{V} \tilde{\mathbf{f}}$ is the scaled vector of inputs to these accumulators (provided by TCM-A from the current state of context), $\mathrm{\kappa}$ is a constant controlling the strength of recurrent inhibition, $\lambda$ is a constant controlling the strength of lateral inhibition, $\mathbf{L}$ is a lateral inhibition matrix with components $L_{i j}=(1-$ $\delta_{i j}$ ), where $\delta_{i j}$ is the Kronecker delta, which equals 1 if $i=j$ and 0 otherwise, $\varepsilon$ is a vector of normally distributed random variables with mean zero and standard deviation $\sigma$, and $\tau$ scales the rate of growth of the accumulators at each time step. The second line reflects a component-wise comparison between $\mathbf{x}_{s}$ and zero such that none of the components of $\mathbf{x}$ can go below zero to prevent negative activations. The equations for the accumulators are exactly as presented in Usher and McClelland (2001), with the exception that rather than a two-choice response, we have $l$ accumulators corresponding to the $l$ items in the list.

The accumulators grow according to Equation 10 until an accumulator crosses a threshold $\Theta$; when this happens, the item corresponding to the accumulator that crosses threshold is recalled. The recalled item is used to update the contextual cue and thus $C_{V}$ and $\tilde{\mathbf{f}}$. Although all accumulators continue to compete across the course of recall, if an accumulator for a recalled item crosses threshold, it is reset to zero without being recalled, thus precluding it from being repeated. ${ }^{8}$

The retrieval process for subsequent recalls. When an item $\mathbf{f}_{i}$ is recalled, TCM-A retrieves a combination of that item's preexperimental context via $\mathbf{M}_{\text {pre }}^{F T}$ as well as the item's experimental context (the state of context when the item was presented) via $\mathbf{M}_{\text {exp }}^{F T}$. These two contextual representations combine by means of Equations 2 and 3 to form the input

\footnotetext{
${ }^{8}$ In free recall of randomly chosen words, healthy young participants very rarely make repetitions (Zaromb et al., 2006); however, a more complete model of recall would need to account for the occasional repetitions that participants do commit.
} 


$$
\mathbf{t}_{i}^{I N}=\left[\left(1-\gamma_{F T}\right) \mathbf{M}_{p r e}^{F T} \mathbf{f}_{i}+\gamma_{F T} \mathbf{M}_{e x p}^{F T} \mathbf{f}_{i}\right]
$$

that contributes to the new state of context calculated via Equation 1.

Depending on the value $\gamma_{F T}, \mathbf{t}_{i}^{I N}$ can be biased toward the newly learned experimental context, which provides a bidirectional cue to items from a similar temporal context, or toward the preexperimental context, which provides a forward-asymmetric cue for words that were studied after the recalled item. Consequently, the bidirectional and forward-asymmetric cues for recall, which give rise to TCM-A's ability to capture contiguity effects in the data, are mediated through associations between items and context and not direct item-to-item associations. Once updated, the new context then serves as the cue for the next recall in the same way that time-of-test context served as the cue for the first recall.

Stopping rule. The criterion for terminating recall is an essential ingredient for modeling serial position effects that was absent from the original TCM implementation. In TCM-A, the accumulators provide a natural stopping rule that is analogous to the time limits imposed on participants in free-recall experiments. Specifically, we assume that recall ends after a fixed number of accumulator time steps. In the subsequent simulations, this value is fixed at 600 time steps.

Modeling distractors. The arithmetic distractors used in delayed and continual distractor free recall are modeled as orthogonal vectors that cause context to drift at a rate $\beta_{\text {dist }}$. Although distractor items drive context, we assume that they do not give rise to any modification of the $\mathbf{M}_{\text {exp }}^{F T}$ and $\mathbf{M}_{\text {exp }}^{T F}$ weight matrices. This assumption seems reasonable, as participants have no reason to learn the distractors. Distractor intervals also differ from item presentations and recalls both in their duration and in the amount and/or type of information being processed. For instance, during a 30 -s distractor interval, participants may be solving approximately 15-20 arithmetic problems, which, in turn, could produce a larger change in context than a single-item presentation.

Modeling primacy. Numerous studies have demonstrated that the two main sources of primacy effects in free recall are an increased tendency to rehearse items from early serial positions throughout the list presentation (Murdock \& Metcalfe, 1978; Rundus, 1971; Tan \& Ward, 2000) and increased attention or decreased competition during the study of early list items giving rise to better encoding (Sederberg et al., 2006). Howard and Kahana (1999) used fast presentation rates and orienting tasks during encoding, which presumably attenuated rehearsal and, in turn, the primacy effect. Still, small primacy effects remained, which often manifested as an increase in the probability of initiating recall with the first item in the list and a higher probability of recalling items from early serial positions, both with respect to middle-list items. Not wanting to complicate TCM-A by including a rehearsal mechanism during encoding, we chose to model primacy as an exponentially decaying boost in the learning rate for early serial positions. This encoding boost is folded into the learning rate $\phi_{i}$ in Equation 5:

$$
\phi_{i}=\phi_{s} e^{-\phi_{d}(i-1)}+1 \text {, }
$$

where $\phi_{s}+1$ determines the size of the primacy effect at the first serial position, and $\phi_{d}$ controls the rate of decay of the primacy effect with each additional list item.

\section{Simulations of Experimental Data}

Table 1 provides a summary of the free and fixed parameters in TCM-A, organized into three categories. The Base category contains the parameters relevant to the theoretical foundation of TCM. The Primacy category lists the parameters specific to our implementation of primacy in the model. Finally, the Accumulator category lists the parameters from the Usher and McClelland (2001) decision model that determines what item is recalled given a set of activations. We allowed the free parameters to vary over a wide range that, based on the equations of the model, encompassed the range of observed behaviors. Similarly, the fixed parameters were selected prior to simulations to be of reasonable values based on the model equations.

Our goal was to produce a reasonable qualitative description of dissociations between short- and long-term recency. This approach has several advantages over attempting to find excellent quantitative fits by separately estimating parameters for each experimental condition being studied. First, it avoids the tendency to overfit the model to certain phenomena, which would prevent the model from simultaneously capturing the trends observed across multiple ex-

Table 1

Summary of Free and Fixed Parameters in TCM-A

\begin{tabular}{llll}
\hline Category & Parameter & \multicolumn{1}{c}{ Description } & Range \\
\hline TCM base & $\beta$ & Rate of contextual drift at item encoding and retrieval. & $0.0-1.0$ \\
& $\beta_{\text {dist }}$ & Rate of contextual drift due to a distractor. & $0.1-1.0$ \\
& $\gamma F T$ & Relative weight of preexp. to exp. context (item-to-context). & $0.0-1.0$ \\
& $\gamma T F$ & Relative weight of preexp. to exp. context (context-to-item). & 0.8 \\
Primacy & $\phi_{s}$ & Primacy scale factor. & $0.0-5.0$ \\
& $\phi_{d}$ & Primacy decay rate. & $0.0-5.0$ \\
Accumulator & $\kappa$ & Strength of recurrent inhibition. & $0.0-0.9$ \\
& $\lambda$ & Strength of lateral inhibition. & $0.0-0.9$ \\
& $\sigma$ & Standard deviation of accumulator noise. & $0.0-0.8$ \\
& $\Theta$ & Recall threshold. & 1.0 \\
& $\tau$ & Rate of growth at each time step. & 0.5 \\
& $\alpha_{\min }$ & Minimum item activation for inclusion in $C_{V}$ scaling. & .0001 \\
\hline
\end{tabular}

Note. Fixed parameters have only a single value, denoted in the Range column. TCM-A $=$ temporal context model-accumulators. 
periments. In addition, it ensures that the model and not the parameters are doing the work in producing the pattern of dissociations observed across multiple experimental conditions.

We used a genetic algorithm (GA) to fit the immediate, delayed, and continual distractor free-recall conditions of Experiments 1 and 2 in Howard and Kahana (1999). The GA starts with a large population of candidate model parameter sets (that span the entire free-parameter space in a multidimensional grid) and, thus, is largely immune to local minima. At each generation, the GA simulates the individual parameter sets and calculates the root-mean-square deviation from the behavioral data. These data include the serial position curve, the probability of first recall, and the contiguity effect as seen in the lag-CRP functions from both the immediate and delayed freerecall conditions of Experiment 1 and from both the delayed and continual distractor free-recall conditions from Experiment 2. The best fitting parameter sets from each generation evolve to form the next generation until reaching a stable state.

We evolved a population made up of 8,000 individuals, which we culled to 1,000 individuals after 50 generations. These 1,000 runs typically took 50 additional generations to stabilize at a single set of parameters that provided approximate fits to all critical conditions. We then performed minor adjustment of the parameters by hand to achieve a better qualitative description of aspects of the data missed by the GA.

\section{Modeling Short-Term and Long-Term Recency}

Our simulation results are organized as follows: First, we fit TCM-A to serial position curves, recency effects, and contiguity effects from immediate, delayed, and continual distractor free-recall tasks reported in Experiments 1 and 2 of Howard and Kahana (1999). Using a single parameterization to fit data from all three tasks and from both experiments, we show that TCM-A also accounts for dissociations between immediate and long-term recency, including the dissociation in the contiguity effect (see Davelaar et al., 2005). Further simulations using the same parameter values account for the differential effect of proactive interference on immediate and longterm recency (Davelaar et al., 2005). Finally, we show that TCM-A can account for the differential effect of amnesia on immediate and long-term recency (Carlesimo et al., 1996).

\section{Simulating Recency and Contiguity in Immediate, Delayed, and Continual Distractor Free Recall}

Our first goal was to assess whether TCM-A could reproduce the basic features of the serial position curve across immediate, delayed, and continual distractor free recall. Figure 3 shows the probability of recall as a function of serial position for the behavioral data and model simulation. TCM-A reproduces the major features of the serial position curve in immediate free recall (see Figure 3, top row, solid line), illustrating a strong recency effect and a modest primacy effect. By simulating a distractor following the study list, TCM-A was able to capture the attenuated recency observed in delayed free recall (see Figure 3, dashed lines). Notably, the difference between immediate and delayed free recall is much greater at the end than at the beginning of the serial position curve. Although the model parameters were fixed between experiments, we plot the delayed condition (dashed line) to provide a point of reference between experiments (top and bottom). In fitting data from Experiment 2, TCM-A captured the
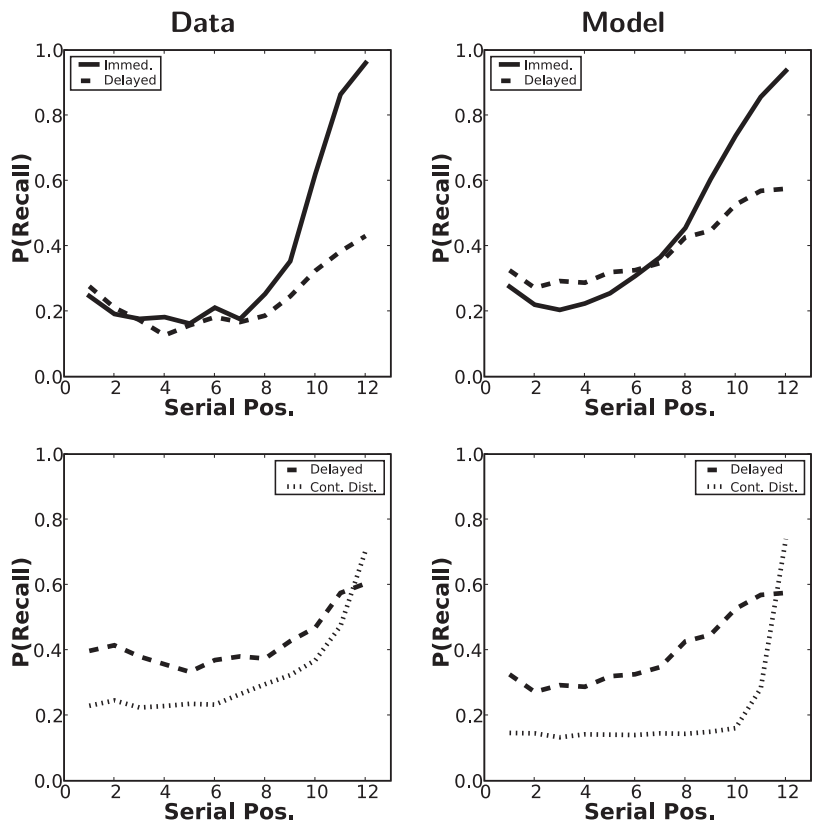

Figure 3. Serial position (Pos.) curves. Behavioral data (left panels) from Howard and Kahana (1999) and model simulations (right panels) of the corresponding free-recall conditions. Immediate (Immed.; solid line) and delayed (dashed line) free recall from Experiment 1 appears in the top row. Delayed (dashed line) and continual distractor (Cont. Dist.; dotted line) free recall from Experiment 2 appears in the bottom row. Note that the simulated fits to the delayed condition (dashed line) are repeated in the top and bottom rows because we did not allow parameters to change between experiments.

relative behavior of the serial position curve in both delayed (see Figure 3, bottom row, dashed line) and continual distractor free recall (see Figure 3, bottom row, dotted line). TCM-A illustrates both the reduced overall level of recall at early serial positions in the continual distractor paradigm and the increased recency effect.

Whereas the serial position curve collapses data over the dynamics of the retrieval process, one can examine separately the way participants initiate recall and the way they make transitions. The probability of first recall (PFR; Hogan, 1975; Laming, 1999), a serial position curve calculated only for the first item recalled, illustrates participants' tendency to initiate recall with one of the terminal list items. As first shown by Deese and Kaufman (1957) the recency effect is closely related to participants' tendency to begin recall at the end of the list (see also Howard \& Kahana, 1999; Kahana, 1996). Figure 4 shows PFR curves generated by the same simulations used to generate the serial position curves in Figure 3. These curves illustrate that participants' tendency to initiate recall at the end of the list was very strong in both immediate and continual distractor free recall but much weaker in delayed free recall. As the PFR functions are strikingly similar in both immediate and continual distractor free recall, this analysis illustrates the persistence of recency across time scales. TCM-A provides a good qualitative fit to both the overall form of the PFR and to the changes in the PFR across distractor schedules.

The foregoing analyses show that TCM-A successfully accounts for the qualitative form of both the serial position and PFR functions across immediate and delayed free recall. TCM-A was also 

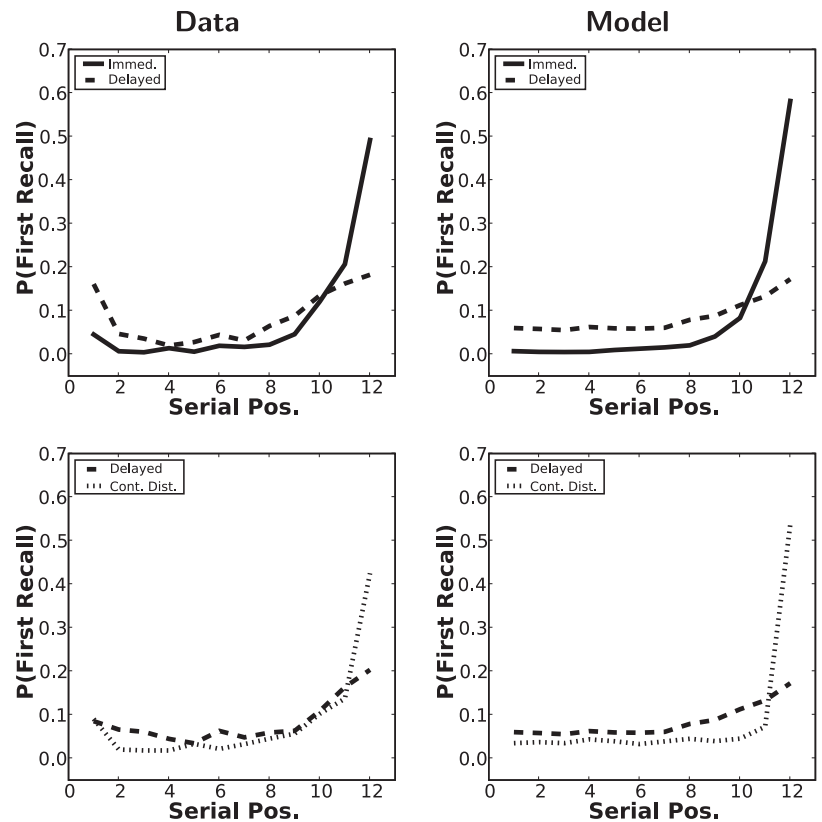

Figure 4. Probability of first recall. Behavioral data (left panels) from Howard and Kahana (1999) and model simulations (right panels) of the corresponding free-recall conditions. Immediate (Immed.; solid line) and delayed (dashed line) free recall from Experiment 1 appears in the top row. Delayed (dashed line) and continual distractor (Cont. Dist.; dotted line) free recall from Experiment 2 appears in the bottom row. Pos. = Position.

able to predict the recovery of recency (both in relative and absolute levels) in continual distractor free recall.

Another striking feature of free recall is the contiguity effect as seen in the lag-CRP analysis of Kahana (1996). Whereas dualstore models account for contiguity on the basis of the cooccurrence of items in STS, contiguity in TCM arises because recall of an item recovers its associated temporal context, which is similar to neighboring list items. Because retrieval is competitive, TCM predicts contiguity across time scales so long as the distractors separating the list items are unrelated to the list items (Howard \& Kahana, 2002a). As shown in Figure 5, the lag-CRP curves generated from the same simulations depicted in Figures 3 and 4
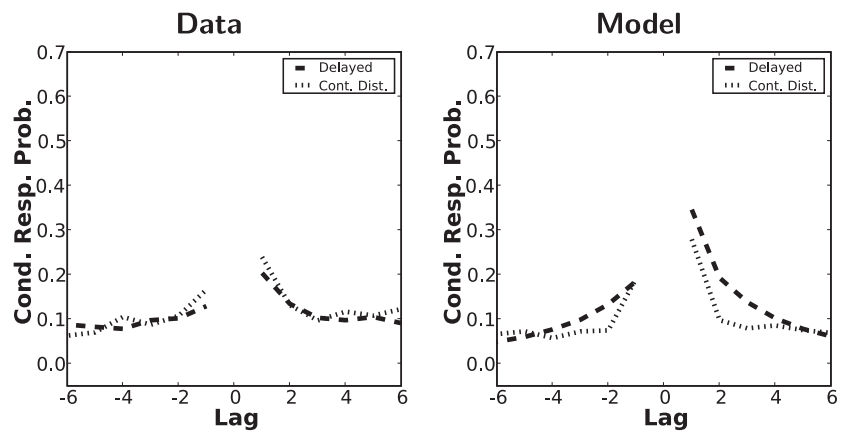

Figure 5. Conditional response probability (Cond. Resp. Prob.). Behavioral data (left panel) from Howard and Kahana (1999) and model simulations (right panel) of delayed (dashed line) and continual distractor (Cont. Dist.; dotted line) free recall from Experiment 2. account for similar levels of contiguity and asymmetry seen in both delayed and continual distractor free recall.

\section{Summary of Parameters}

Table 2 illustrates the model parameters (eight free) that produced the fits shown in Figures 3, 4, and 5. The contextual drift rate during item presentations and retrievals $(\beta)$ is moderately fast, which gives rise to the sharp contiguity effect observed in Figure 5. Similarly, the contextual drift during a distractor interval $\left(\beta_{\text {dist }}\right)$ is quite large, meaning that the distractor interval, which may contain multiple math problems, has as large an effect on the previous context vector as several item presentations would have. The value of $\gamma_{F T}$ indicates that the relative contributions of preexperimental and experimental item-to-context associations are nearly equivalent (there is a slight bias toward preexperimental context). The scale and decay of primacy $\left(\phi_{s}\right.$ and $\left.\phi_{d}\right)$ provide a strong boost for the first item in the list, but this boost decays quite quickly for subsequent serial positions, dropping from approximately 2.66 for the first item to approximately 1.23 for the second item, which is close to the asymptotic value of 1 . For the accumulator, we required the lateral inhibition $\lambda$ to be equal to the leak (decay) rate $\kappa$, resulting in accumulators that follow a drift diffusion process (Ratcliff, 1978); this has been shown to be desirable when fitting reaction times of decision processes (Bogacz, Brown, Moehlis, Holmes, \& Cohen, 2006; McMillen \& Holmes, 2006; Smith \& Ratcliff, 2004). Finally, the relatively high variance of the accumulator's noise parameter enables remote transitions during recall, as this is the only source of variability included in our simulations.

\section{Dissociations Between Short- and Long-Term Recency}

The preceding simulations demonstrate that TCM-A can explain the basic recency and contiguity phenomena across conditions with a single set of parameters. In particular, TCM-A is able to describe the commonalities between short- and long-term recency and the analogous similarity between contiguity effects observed in delayed and continual distractor free recall, both of which are expressed in relative probabilities of recall. Using the same model, and the same set of parameters, we now demonstrate that TCM-A

Table 2

Best Fitting Parameters for TCM-A Simulations

\begin{tabular}{lcl}
\hline Category & Parameter & \multicolumn{1}{c}{ Value } \\
\hline TCM base & $\beta$ & 0.62676 \\
& $\beta_{\text {dist }}$ & 0.97607 \\
& $\gamma F T$ & $0.44542(0.0)$ \\
Primacy & $\gamma T F$ & $0.8(0.0)^{*}$ \\
& $\phi_{s}$ & 1.65538 \\
Accumulator & $\phi_{d}$ & 1.98112 \\
& $\kappa$ & 0.62000 \\
& $\lambda$ & 0.62000 \\
& $\sigma$ & $0.29934(0.28284)$ \\
& $\Theta$ & $1.0^{*}$ \\
& $\tau$ & $0.5^{*}$ \\
\hline
\end{tabular}

Note. Parameter values marked with an asterisk were fixed. Parameter values in parentheses were used for the amnesic simulations. TCM-A = temporal context model-accumulators. 
is also able to account for the major dissociations between shortand long-term recency. The following sections demonstrate TCMA's ability to capture these dissociations using gradually changing temporal context as the sole retrieval cue to initiate recall across conditions. In fact, temporal context, governed by the equations described above, is the sole retrieval cue at all recall attempts.

Time to first recall. Although it has not been explicitly reported before, it is well-known to investigators examining immediate and continual distractor free recall that the recall latency to the first response differs across these conditions. Figure 6 illustrates the model's ability to capture this dissociation with the latencies to initiate recall in the immediate, delayed, and continual distractor free-recall conditions for both the behavioral data from Howard and Kahana (1999) and the simulations. Although the immediate and continual distractor free-recall data are taken from different experiments, the delayed free-recall conditions from Experiments 1 and 2 of Howard and Kahana (1999) provide a reference point for the comparison. The fact that the delayed free-recall conditions exhibit comparable response times suggests that there is a meaningful difference between latencies in the immediate and continual distractor free-recall conditions.

This finding reflects a key advantage of TCM-A. The accumulators are sensitive to both the relative activations of the list items and to their absolute level of activation. In particular, the greater overall level of activation for end-of-list items in immediate free recall causes the accumulators to reach threshold more quickly than in continual distractor free recall. Note that if there is a limited amount of time for recall, then faster recall latencies translate into a higher probability that some item is recalled within the time limit.

Contiguity effects in early output positions. The contiguity effect in immediate free recall is much stronger at the first couple of output positions than it is later in recall (Kahana, 1996). This change in the shape of the lag-CRP with output position is not observed in either delayed or continual distractor free recall (Howard \& Kahana, 1999). Figure 7 demonstrates that TCM-A

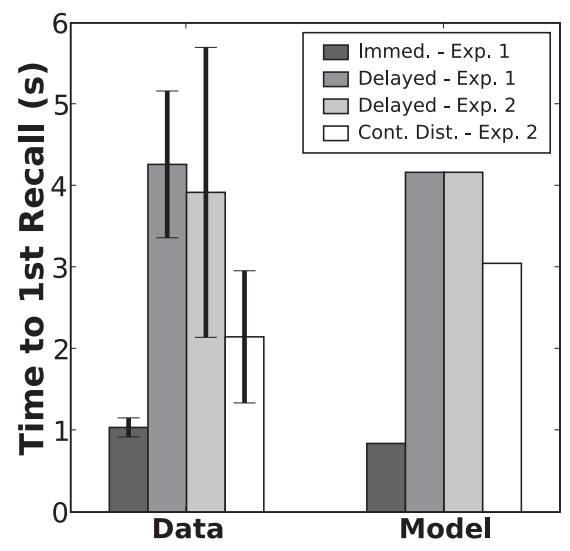

Figure 6. Time to first recall. Data and model fits of the time, in seconds, until the first recalled item. White bars indicate the time to the first recall in continual distractor (Cont. Dist.) free recall, whereas dark gray bars provide the time to the first recall in immediate (Immed.) free recall. The intermediate grey bars show the time to first recall in the delayed freerecall conditions of Experiments 1 and 2 from Howard and Kahana (1999), as a reference. Note that model output is in time steps, scaled by 40 to match the behavioral data. Error bars are $95 \%$ confidence intervals.
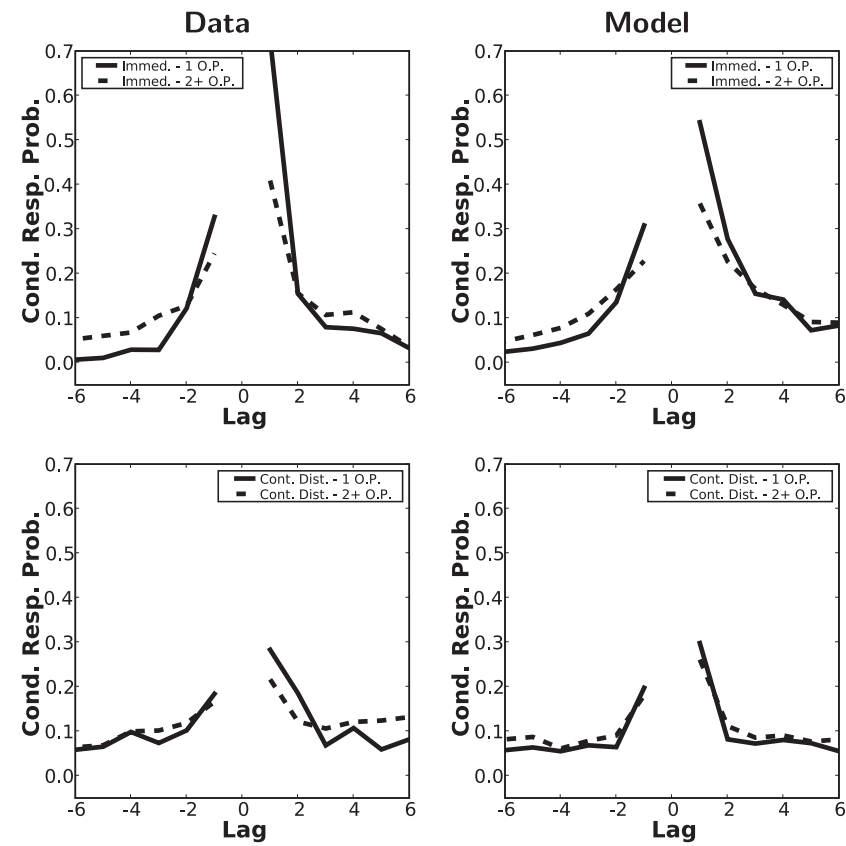

Figure 7. Conditional response probabilty (Cond. Resp. Prob.) by output position (O.P.). Lag-CRPs of the first (solid line) and then remaining (dashed line) output transitions for behavioral data (left panels) from Howard and Kahana (1999) and model simulations (right panels) in the immediate (Immed.) free-recall condition of Experiement 1 (top panel) and the continual distractor (Cont. Dist.) free-recall condition of Experiment 2 (bottom panel).

can account for the decline in the contiguity effect across output positions seen in immediate free recall and the lack of such a change in the contiguity effect across output positions in continual distractor free recall. The top row of Figure 7 shows that TCM-A captures the reduction in the contiguity effect between the first output position (solid line) and later output positions (dashed line) in immediate free recall. The bottom portion of Figure 7 shows that TCM-A predicts correctly the absence of any change in the contiguity effect across output positions in continual distractor free recall. The dissociation was observed in the same simulations that generated the serial position curves, PFRs and lag-CRPs, above.

Proactive interference. Davelaar et al. (2005) showed that although the short-term recency effect, as measured by the probability of recall of end-of-list items, was not sensitive to proactive interference, the long-term recency effect was. We examined whether TCM-A can account for the dissociation between the effect of proactive interference on short- and long-term recency by re-running the simulations reported above with lists of varying length. The items at the end of the longer lists are subject to more proactive interference from preceding list items than items at the end of shorter lists. As illustrated in Figure 8, TCM-A predicts correctly that the probability of recall for the recency part of the serial position curve remains relatively constant in immediate free recall (left panel) but declines with the same increase in list length in continual distractor free recall (right panel).

Anterograde amnesia. Carlesimo et al. (1996) examined the effect of anterograde amnesia on both immediate and continual distractor free recall. Both amnesics and controls exhibited strong 

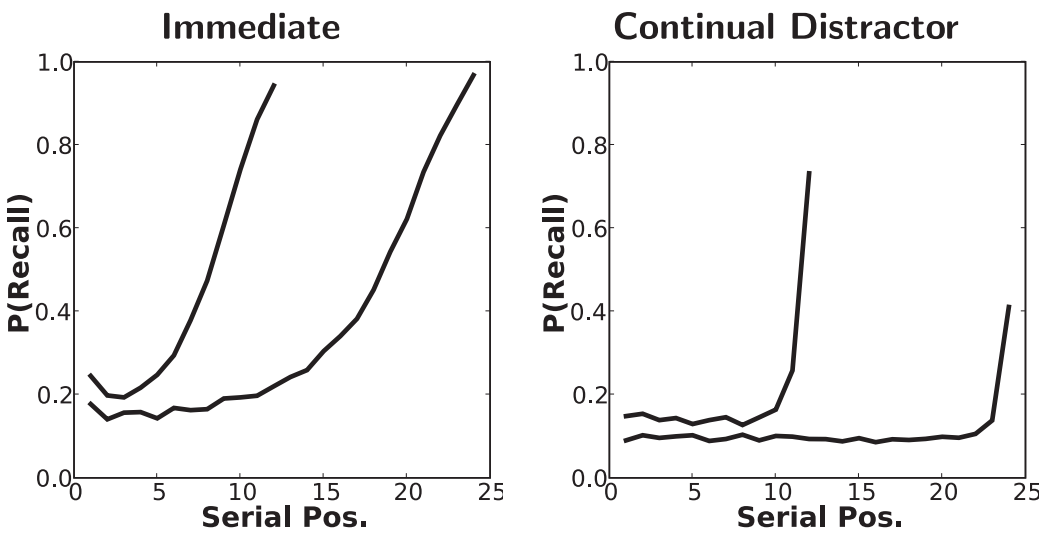

Figure 8. Proactive interference effect. Model simulations of serial position (Pos.) curves illustrating the effect of increasing the list length in immediate free recall (left panel) versus continual distractor free recall (right panel).

recency effects in both tasks. Although amnesics recalled substantially fewer words in both tasks, their recall of recency items was comparable to that of controls in immediate free recall. In continual distractor free recall, however, amnesics recalled fewer words than controls at all serial positions.

We hypothesize that the effect of anterograde amnesia on memory can be described by a disruption in the ability to associate an item with its temporal context and for an item to recover its temporal context when repeated. In other words, we assumed that no learning took place at $\boldsymbol{M}_{\text {exp }}^{F T}$ and $\boldsymbol{M}_{\text {exp }}^{T F}$ (i.e., $\gamma T F=\gamma F T=0$ ) for our simulated amnesia patients. In addition, we assumed that the accumulator-based retrieval process would exhibit less variability in amnesics than in controls. Decreasing the variability of the accumulators (i.e., reducing the standard deviation of the noise, $\sigma$, in Equation 10) reduces the probability of spontaneous fluctuations above threshold in the absence of a real memory signal. Consequently, it will take longer, on average, for any item to jump above the recall threshold for amnesics. The assumption that amnesics exhibit decreased accumulator noise relative to controls makes sense if one assumes that at least some of the noise is the consequence of variability in the strength of the memory signal. Because we have not yet expanded TCM-A to include variability in itemto-context or context-to-item associations, or in the structural similarity among items in semantic memory, the stochastic nature of the retrieval process is the only source of variability in our model. As such, increasing variability in the accumulators is functionally analogous to increasing variability in goodness-ofencoding or semantic structure within the list. In the General Discussion section, we discuss enhancements to TCM-A that include encoding variability and preexperimental semantic associations between items, either of which might account for the explicit noise decrease in our simulations.

Besides the changes to the learning and variability parameters, all other aspects of the model-the gradual change of temporal context from moment to moment, the preexperimental weights (i.e., $\boldsymbol{M}_{\text {pre }}^{F T}$ and $\boldsymbol{M}_{\text {pre }}^{T F}$ ), and the retrieval dynamics of the accumulators - were left intact. Notably, we hypothesize that the rate of contextual drift, parameterized by $\beta$, will be unchanged in amnesia patients. The equivalence of the rate of contextual drift, coupled with intact preexperimental context-to-item associations, means that there will be little to no difference in the effectiveness of the test context as a cue for recall between amnesics and controls. As a result, amnesics should exhibit recency effects that are similar to that of controls in both immediate and continual distractor free recall.

In contrast, our model predicts that contiguity effects are dependent on contextual retrieval and the formation of new context-to-item associations. Because these processes are hypothesized to be disrupted in amnesics, this predicts that the lag-CRP should be flat for amnesics in both continual distractor free recall and at later output positions in immediate free recall. Our account of short-term recency exploits the fact that the accumulators are sensitive to the absolute magnitude of activation, allowing the strong end-of-list cue available in immediate recall to cause the recall of several recency items. This immediate recall of multiple recency items results in a boost to the contiguity effect at early output positions in immediate free recall. Because contextual drift is unchanged in amnesics, the time-of-test context is identical to that of controls. Given that multiple recency items are strongly represented in the end-of-list context in immediate free recall, this will give rise to a boost in the probability of recalling more than one item from the end of the list at the start of recall. Thus, even in the absence of contextual retrieval, we expect that amnesics would also exhibit a peaked contiguity effect at early output positions in immediate recall.

Although probability of first recall and lag-CRP curves are not available for the Carlesimo et al. (1996) study, we generated them for our simulated amnesics. Because we are not attempting a quantitative fit of the Carlesimo et al. (1996) data, we have left all experimental parameters (list length, delay intervals, etc.) unchanged from our simulations of the Howard and Kahana (1999) data. The curves shown for simulated control subjects are identical to those shown in Figures 3 and 4.

As can be seen by comparing the top-left and top-right panels of Figure 9, TCM-A predicts correctly that the overall level of recall drops for amnesics in both immediate and continual distractor free recall. Although the recency effect, as measured by the PFR curves in the bottom panels of Figure 9, is nearly identical for controls and amnesics in both immediate and continual distractor free recall, the probability of recalling the last item in the list decreases only in the continual distractor simulations. As outlined above, this pattern of results is consistent with previous studies (Carlesimo et al., 1996; 

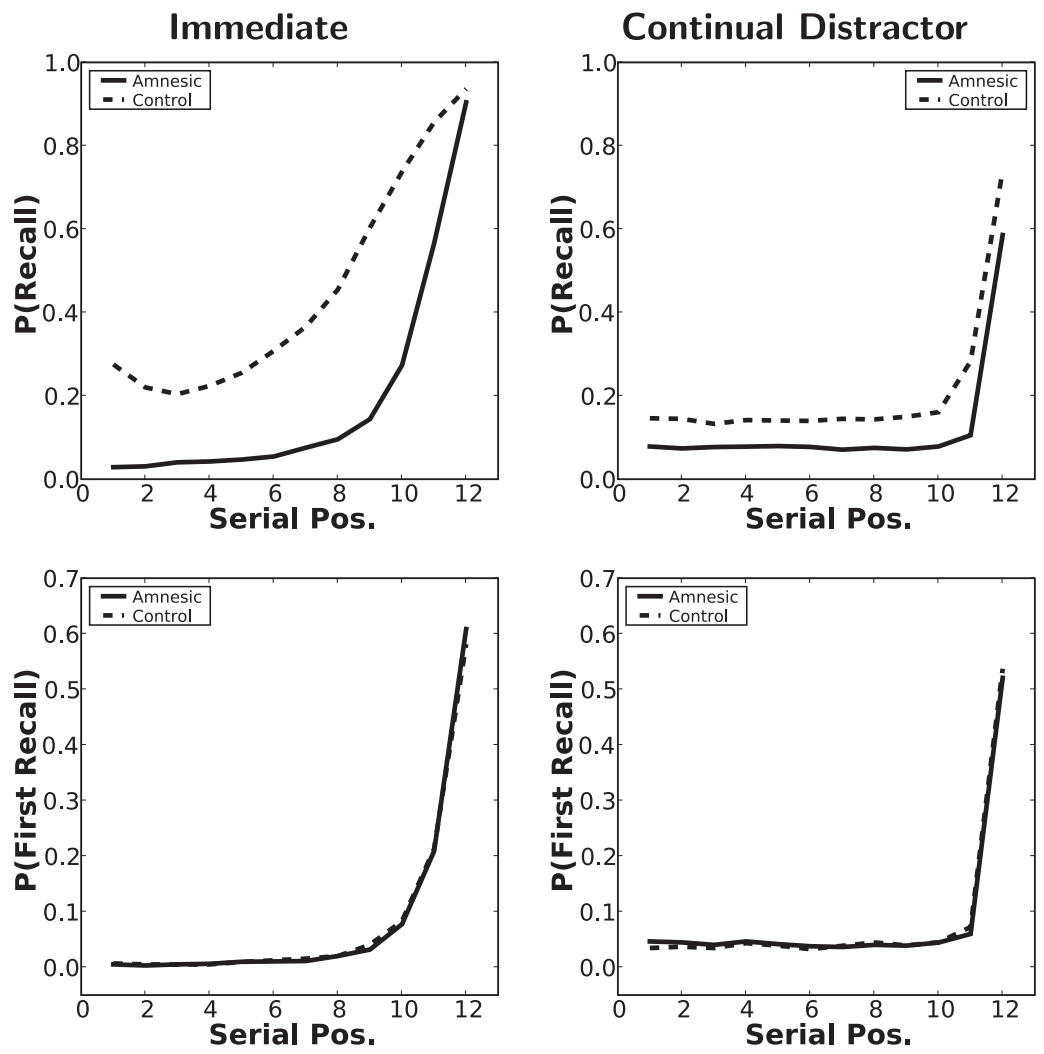

Figure 9. Effects of amnesia on probability of recall. Serial position (Pos.) curves (top panel) and probability of first recall (bottom panel) showing simulated effects of amnesia (solid lines) versus controls (dashed lines) in immediate free recall (left panels) and continual distractor free recall (right panels).

Marks \& Cermak, 1998), which have shown that recency persists in amnesic participants but that the overall probability of recall drops at all serial positions in continual distractor free recall.

In contrast with the predictions for both overall recall levels and the probability of first recall, TCM-A produced dramatic differences in the lag-CRP in the simulated amnesics across distractor conditions. The simulated amnesics showed a peaked lag-CRP at the first output position in immediate free recall but a flat lag-CRP at later output positions (see Figure 10, left panel). In contrast, the contiguity effect persisted across output positions in the simulated control participants (see Figure 7, top-right). In continual distractor free recall, the simulated amnesics showed a flat lag-CRP both early and late in output.

The fact that our amnesia simulations resulted in a peaked lag-CRP early in immediate but not in continual distractor free recall reflects an important dissociation between short- and long-term recency. The greater absolute levels of activation resulting from end-of-list context in immediate free recall is sufficient to drive recall of several end-oflist items. In contrast, the absolute level of activation due to end-of-list context in continual distractor free recall is relatively weaker, such that it does not affect multiple retrieval attempts.

\section{Essential Properties of the Retrieval Rule}

TCM-A's ability to capture the dissociations between short- and long-term recency results from the retrieval rule generating several fast recalls from the end of the list in response to the strong end-of-list cue in immediate free recall. Here we describe how our parameterization of TCM-A produces the dissociations described above.

Figure 11 displays the input to the accumulators $\left(C_{V} \tilde{\mathbf{f}}\right)$ derived from the time-of-test context for each item as a function of serial position (see Equations 9 and 10). The inputs in both immediate and continual distractor free recall show a strong recency effect. However, the absolute magnitude of the inputs is smaller in the continual distractor condition. This difference in magnitude is due to the distractor delay in continual distractor free recall, which gives rise to a smaller overlap between the time-of-test context and the encoding context of the list items (Howard, 2004). ${ }^{9}$ The overall scale of the accumulator growth due to the input is largely determined by the standard deviation of the noise term, $\sigma$, which took on a value of approximately 0.3 in our simulations (see Table 2). Given that the only sources of accumulator growth are the input and the noise (see Equation 10), if the noise is much larger than the input, then it will mask the effect of the input. Inspection of

\footnotetext{
9 The inclusion of the coefficient of variation in Equation 10 counteracts this difference in the absolute level of activation. However, the inclusion of the threshold $\alpha_{\min }$ causes the coefficient of variation in continual distractor free recall to be larger than it would be without the threshold, allowing the model to preserve the difference in overall activation between immediate and continual distractor free recall.
} 
Figure 11 shows that although the activation of several items is near or above $\sigma$ when immediate recall is initiated, only the last item in the list has an activation on the order of $\sigma$ in continual distractor free recall.

The absolute difference in the level of activation at the initiation of immediate compared with continual distractor free recall leads to the predicted differences between the short- and long-term recency effects. For instance, the greater magnitude of the activations in immediate free recall means that the accumulators reach threshold more quickly, accounting for the difference in time to initiate recall. Critically, the greater absolute level of activation of several end-of-list items in immediate free recall enables TCM-A to recall several end-of-list items at the start of the retrieval period. Although context is assumed to evolve during retrieval as well as during list presentation, the strong end-of-list cue that is present when immediate recall is initiated persists, weighted by $\rho$, as part of the cue for the second recall. Consequently, the strong residual activation from the end-of-the list items in immediate free recall combines with the reinstated context to provide a stronger cue for recall than retrieved context would alone. Thus, the average input to the accumulators for the second output in immediate free recall is still higher for items from the end of the list than for other items, giving rise to the enhanced recall probability for end-of-list items.

For the parameters used in these simulations, residual activation in the accumulators between responses is not contributing to the recency dissociations. The original motivation for using the accumulators in TCM-A was the expectation that nonrecalled recency items in immediate free recall would have an increased probability of being recalled in quick succession because they would all be close to threshold when any one of them was recalled. However, for the parameters used in these simulations, resetting all the accumulators to zero following each recall had no effect on the simulated results. ${ }^{10}$ The dissociations between short- and longterm recency observed in the simulations thus do not depend on persistent activation of items across retrieval attempts but rather reflect properties inherited from the rules for contextual evolution and recovery provided by TCM.
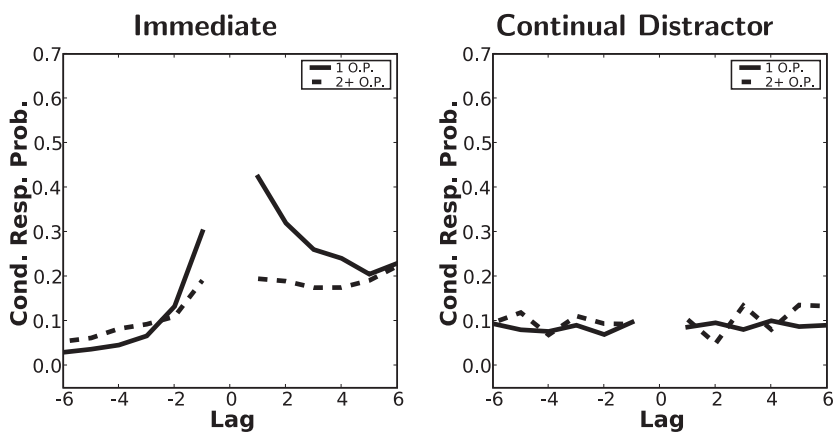

Figure 10. Effects of amnesia on conditional response probability (Cond. Resp. Probl). Conditional response probability by output position (O.P.) curves showing simulated effects of amnesia in immediate free recall (left panel) and continual distractor free recall (right panel). Just as in Figure 7, these panels compare the lag-CRP from the first output position (solid lines) with all other output positions (dashed lines), but for the simulated amnesics.

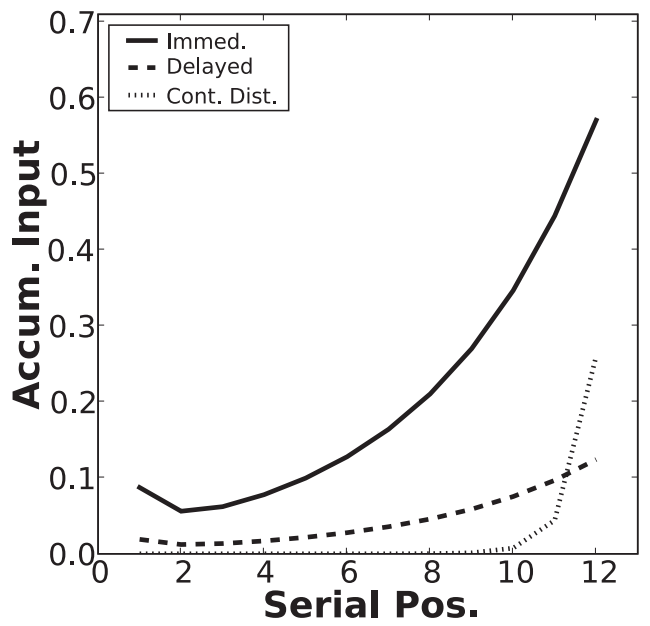

Figure 11. Accumulator (Accum.) input as a function of serial position (Pos.). Accumulator input for immediate (Immed.), delayed, and continual distractor (Cont. Dist.) free-recall conditions for the first output position.

\section{General Discussion}

The TCM-A model assumes that a gradually changing context representation serves as the sole retrieval cue throughout immediate, delayed, and continual distractor free recall. We have shown how TCM-A not only captures the standard serial position, recency, and contiguity effects (see Figures 3-5) but also accounts for several key dissociations between short- and long-term recency. These dissociations include the findings of faster recall initiation in immediate as compared with continual distractor free recall (see Figure 6), more local recall transitions among recently studied items in immediate than in continual distractor free recall (see Figure 7), significantly greater proactive interference in continual distractor than in immediate free recall (see Figure 8), and differing effects of anterograde amnesia on immediate and continual distractor free recall (see Figures 9 and 10).

The gradually changing context representation in TCM-A cues recall of items using a combination of preexperimental (e.g., $\boldsymbol{M}_{\text {pre }}^{T F}$ ) and newly learned (e.g., $\boldsymbol{M}_{\text {exp }}^{T F}$ ) weight-based memories. Due to the temporal evolution of this context representation, and its associations with the studied items, recent items are activated more than remote items when cued with time-of-test context. This relative advantage for recent items gives rise to the recency effect observed both in immediate and continual distractor free-recall tasks. Although the relative activations of recent as compared with remote items depends primarily on the relative timing of item presentations (which is constant across immediate and continual distractor free recall), the absolute magnitude of the activations is substantially greater in immediate than in continual distractor free recall. It is this difference in absolute activations that enables TCM-A to account for the dissociations between immediate and long-term recency.

\footnotetext{
${ }^{10}$ It is also possible that allowing the accumulator for the just-recalled item to evolve during retrieval is affecting the behavior of the model by means of lateral inhibition.
} 


\section{The Associative Framework of TCM-A}

Following study or recall of an item, the retrieval of preexperimentally learned and newly learned associations combine to update the current state of context. During recall, the current state of context acts as the sole cue for retrieval of its associated item representations. As items are presented for study, TCM-A stores new experimental associations between items and context, but preexperimental associations are not updated. Although these encoding and retrieval processes have separate components, it is overly simplistic to think of any piece in isolation as a store that holds memory traces.

Consider what would happen if we were to follow the process of learning far beyond the time scale of a single list. In this case, learning of item-to-context and context-to-item associations would build up over time to describe the set of contexts in which an item has been presented. However, at some point, it would seem necessary for the labile "experimental" component of these associative matrices to become the fixed "preexperimental" component of the associative matrices. This would allow the model to account for the finding that in amnesics, some "long-term memories" are intact, although the formation of new long-term memories is impaired.

In this sense, the associative framework of TCM-A may fit well with the theory of complementary learning systems, which posits that there are fast and slow-learning mechanisms in the brain, working in concert to learn and store information (McClelland, McNaughton, \& O'Reilly, 1995). The fast system, which is composed of the hippocampus and other medial temporal lobe structures, is able to learn associations quickly but is unable to store these representations for long periods of time. Over time, learned associations are transferred to the cortical system, which learns slowly but holds lasting representations of a person's experience. This type of model (see also Alvarez \& Squire, 1994) has often been proposed to account for the standard model of consolidation in amnesia (Eichenbaum, Otto, \& Cohen, 1994; Squire, 1992; but see Nadel \& Moscovitch, 1997, 2001).

\section{Short-Term Memory and Temporal Context}

The equation describing the change of context from moment to moment in TCM-A (Equation 1) is reminiscent of the correlated contextual fluctuations of variable context models (Anderson \& Bower, 1972; Mensink \& Raaijmakers, 1988; Murdock, 1997). These correlated vector states can be seen as an activation-based memory in the sense Davelaar et al. (2005) used the term. Indeed, Howard, Fotedar, Datey, and Hasselmo (2005) noted that a very close analogue of Equation 1, with persistent neural activity representing the current state of context, could be implemented in a neurally realistic simulation that relied on known properties of cells in the entorhinal cortex (Egorov, Hamam, Fransen, Hasselmo, \& Alonso, 2002; Fransén, Tahvildari, Egorov, Hasselmo, \& Alonso, 2006) and cortical networks (Chance \& Abbott, 2000; Chance, Abbott, \& Reyes, 2002).

In TCM-A, fluctuations in temporal context are not random, but are caused by the sequence of presented and recalled items and the retrieval of their associated contexts. Thus, one might view context as being conceptually analogous to an activation-based memory. To see why this is so, consider what would happen if contextual learning were turned off (mathematically, suppose $\gamma F T=0$ ) so that each time an item is presented it causes the same pattern of input to the temporal context vector. Because there is a one-to-one correspondence between this input pattern and the item being presented, these patterns can be thought of as a representation of the item itself. In this case, the temporal context vector contains a weighted sum of the patterns of recently presented items.

There are two salient differences between $\mathbf{t}_{i}$ and traditional buffer models. One difference is that in TCM-A, the input patterns do not drop out in an all-or-none fashion but decay gradually as more information comes in. This property enables TCM-A to describe recency and contiguity effects over different time scales with a single retrieval mechanism (see also Howard \& Kahana, 2002a). The other salient difference is that in TCM-A, the patterns caused by an item do not, in general, stay constant over multiple repetitions of the item, but rather change to reflect the changing contexts in which the item has been presented. It is this latter property that enables TCM-A to describe contiguity effects (see also Howard et al., 2006), including those observed in short-term recency (Howard, Vankatadass, Norman, \& Kahana, 2007). Viewed in this light, the distinction between a temporal context vector and a traditional STS is not so much about a fundamental distinction between activity-based storage versus something else, but rather about the way activity-based storage actually works. Of course this is not to suggest that these particular distinctions do not have important implications for how researchers understand memory but that they do not have a direct bearing on the question of whether activity-based storage contributes to the recency effect. Indeed, TCM-A and traditional buffer models make distinct predictions about how memory should behave at both short- and long-time scales. In the next section, we review some of the evidence in support of contextual-based memory from both behavioral and electrophysiological studies.

\section{Evaluating Predictions of Temporal Context Across Time Scales}

Howard et al. (2007) compared behavioral predictions of TCM-A with those of buffer models in describing associative effects in the early stages of the immediate recency effect. In this study, some lists contained an item from the middle of the list that was repeated just before the test. Howard et al. (2007) observed a boost for neighbors of the original presentation of the repeated item in early recall transitions, and even in the initiation of immediate recall. Although TCM-A predicts these associative effects as a natural outcome of using temporal context as the cue to initiate immediate recall, buffer models of immediate recency would predict no such effect. One would not expect the predictions of TCM-A about immediate recency to be borne out over those of a buffer model if gradually changing temporal context was not the cue for the initiation of immediate free recall.

Whereas Howard et al. (2007) confirmed behavioral predictions of TCM over a few seconds, Howard et al. (2008) evaluated behavioral predictions over a much longer time scale. Participants were presented with 48 lists one at a time for immediate free recall. At the end of the session, they were given a surprise final freerecall test in which they were instructed to recall as many items as possible from all the lists in any order they came to mind. In examining the final free-recall data, Howard et al. observed a 
recency effect that extended six to eight lists into the past. Moreover, Howard et al. observed a contiguity effect across lists in the final free-recall data that extended about 10 lists in each direction. A particularly striking feature of these data was the similar functional form observed for the recency and contiguity effects across time scales, suggesting that a similar cuing mechanism operates over short and long time scales, as predicted by TCM-A.

TCM-A naturally explains the findings of Howard et al. (2008) if the temporal context vector is allowed to change gradually across lists in addition to changing gradually within each list. If the temporal context vector is an activation-based memory in the medial temporal lobe, as hypothesized by Howard et al. (2005), then this means that there should be neural activity in the medial temporal lobe that persists across lists.

Manns, Howard, and Eichenbaum (2007) examined the ensemble firing patterns of cells in the hippocampus while rats performed a judgment-of-recency task on lists of odors. In their experiment, rats were given "lists" of five odors. At the end of each list, the rats were presented with two odors from the list and rewarded for approaching the one that was presented earlier in the list. The rats were implanted with electrodes in the hippocampus that were able to record simultaneously from multiple, as many as several dozen, hippocampal neurons. Manns et al. (2007) treated these ensembles as vectors of neural activity and examined the way the ensemble firing pattern changed across time periods when the rat was "studying" the list odors. Manns et al. (2007) showed that the neural activity vector was more similar for study events that occurred closer together in time than for events that occurred further apart in time (see Equation 1). That is, all other things being equal, the ensemble patterns for study events corresponding to nearby serial positions within a particular list were more similar to each other than ensemble patterns corresponding to study events from serial positions farther apart in the list. Remarkably, Manns et al. (2007) also observed that the neural ensemble response changed gradually across lists as well, with measurable changes in the ensemble vector extending over essentially the entire recording session. This long-range correlation observed in the neural activity vector across lists in the rat hippocampus parallels the long-range correlations predicted for the temporal context vector across lists of words based on behavioral results from humans (Howard et al., 2008). These recent findings suggest that the TCM framework captures some basic insights about how episodic memory is encoded and retrieved at both short and long time scales.

\section{Ingredients for a More Complete Model of Free Recall}

Although TCM-A can account for many of the major findings in free recall, the model is still missing a number of mechanisms that would be required to explain the full range of benchmark phenomena. Specifically, TCM-A does not have the necessary machinery to explain semantic similarity, category clustering, and falsememory effects. TCM-A is also missing a realistic model of encoding and rehearsal processes, which have been shown to be related to several important recall phenomena such as the primacy effect. Below we discuss potential extensions of TCM-A that would make it a more complete description of free recall.

Semantic retrieval effects. Semantic relations between words have been demonstrated to influence behavior during memory tasks, giving rise to category clustering (Bousfield, 1953; Shuell,
1969) and semantic proximity effects (e.g., Howard \& Kahana, 2002b; Romney, Brewer, \& Batchelder, 1993). Computational models of semantic structure have exploited the fact that words with similar meaning occur in similar temporal contexts (Griffiths, Steyvers, \& Tenenbaum, 2007; Jones \& Mewhort, 2007; Landauer \& Dumais, 1997; Lund \& Burgess, 1996).

Zaromb et al. (2006) demonstrated that participants' prior list intrusions - incorrect recalls of items from earlier lists-tend to be semantically related to the correct recall that occurred just before the intrusion. This finding in recall of random word lists is reminiscent of the more striking demonstrations of false recall when lists are composed of items that are all strong associates of a critical nonpresented item, as in the Deese-Roediger-McDermott false-memory paradigm (Deese, 1959; Kimball et al., 2007; Roediger \& McDermott, 1995). The TCM framework would suggest that prior list intrusions arise due to an interaction between the slowly drifting temporal context cue and semantic associations between items as expressed in the item-to-context and context-toitem matrices. In other words, the current state of temporal context would weakly activate items in prior lists to the extent that the items occurred recently and would provide an additional boost to items that are semantically similar to the current context.

On the basis of these findings, one possible way to include semantic associations in the TCM framework is to have offdiagonal terms of the preexperimental context-to-item association matrix code for the semantic representation of an item. This could allow for contextually mediated semantic retrieval effects such as prior list intrusions and false memory.

Encoding variability. Presently, the only source of variability in TCM-A is the stochastic noise in the accumulators. Although having a single source of variability does simplify analysis of the model, this is clearly not the case for actual memory. Numerous studies have reported that variability in brain activity during item encoding predicts what items will be recalled later (Paller \& Wagner, 2002; Sederberg, Kahana, Howard, Donner, \& Madsen, 2003; Sederberg, Schulze-Bonhage, Madsen, Bromfield, Litt, et al., 2007; Sederberg, Schulze-Bonhage, Madsen, Bromfield, McCarthy, 2007). Such subsequent memory effects suggest that the level of encoding varies during the study list and that this affects subsequent memorability. In TCM-A, encoding variability could be implemented by treating $\gamma T F$ and/or $\gamma F T$ as a random variable that varies from item to item. ${ }^{11}$ In other words, the weight of newly learned experimental item-to-context associations would change for each item presentation. Theoretically, it should be possible to set the degree of encoding on the basis of neural activity recorded from participants performing the task, thus providing a link between the computational theory of episodic memory and brain data.

Rehearsal. Rehearsal processes have been implicated in a number of free-recall phenomena, including primacy effects and spacing effects (e.g., Rundus, 1971). Rehearsal could be implemented in TCM-A by allowing item retrievals during study, with recalled items receiving an encoding boost. This would allow for a direct comparison with overt rehearsal paradigms and the relationship between rehearsals and recalls (Laming, 2006; see also

\footnotetext{
${ }^{11}$ It is also possible that the encoding of item-to-context and contextto-item associations are correlated across trials.
} 
Tan \& Ward, 2000) as well as provide an explanation for why primacy effects are greatly diminished in studies in which rehearsal is limited (Howard \& Kahana, 1999). Given that rehearsals would follow the same process as retrievals in TCM-A, it may be possible to map primacy effects to neural mechanisms as well.

Going beyond temporal context. In the present implementation of TCM-A, temporal context evolves solely as a result of items being activated during presentation or retrieval. Although this appears to be adequate for simulating encoding and retrieval of a standard free-recall list, items alone do not reflect the rich temporal context that people experience while encoding new experiences, even while in a controlled lab environment. One could envision an extension of TCM-A whereby additional sources of context would be represented in the model. Other contextual features could include the participants' task during encoding; the modality or source information of each item; ordinal information; list information; spatial information, including the location of a word on the screen and the spatial environment of the participant during the experiment; and even the participant's affective state. An extension of TCM-A in which this broader view of context is incorporated might allow the model to account for a far richer array of cognitive phenomena.

\section{Conclusions}

We have shown how TCM-A-a contextual-cuing model of episodic memory - can account for a wide range of recall phenomena, including recency and contiguity effects in immediate, delayed, and continual distractor free recall. In addition, TCM-A was able to account for a number of major empirical dissociations between recency effects observed in immediate and continual distractor free recall, including the longer time to first recall in continual distractor free recall, changes in the contiguity effect with output position in immediate free recall, as well as the resistance of recency in immediate free recall to both proactive interference and amnesia. The ability to account for these dissociations is striking because the model assumes that the same retrieval process supports recency and contiguity in both shortterm and long-term memory tasks. Thus, we would argue that the universal phenomenon of recency in memory tasks may reflect a single basic forgetting mechanism, and that mechanism may be the fluctuating internal contextual state of the organism (e.g., Estes, 1950; McGeoch, 1932).

\section{References}

Alvarez, P., \& Squire, L. R. (1994). Memory consolidation and the medial temporal lobe: A simple network model. Proceedings of the National Academy of Sciences, USA, 91, 7041-7045.

Anderson, J. R., \& Bower, G. H. (1972). Recognition and retrieval processes in free recall. Psychological Review, 79, 97-123.

Atkinson, R. C., \& Shiffrin, R. M. (1968). Human memory: A proposed system and its control processes. In K. W. Spence \& J. T. Spence (Eds.), The psychology of learning and motivation (Vol. 2, p. 89-105). New York: Academic Press.

Bjork, R. A., \& Whitten, W. B. (1974). Recency-sensitive retrieval processes in long-term free recall. Cognitive Psychology, 6, 173-189.

Bogacz, R. (2007). Optimal decision-making theories: Linking neurobiology with behaviour. Trends in Cognitive Sciences, 11, 118-125.

Bogacz, R., Brown, E., Moehlis, J., Holmes, P., \& Cohen, J. D. (2006). The physics of optimal decision making: A formal analysis of models of performance in two-alternative forced-choice tasks. Psychological Review, 113, 700-765.

Bogacz, R., Usher, M., Zhang, J., \& McClelland, J. L. (2007, April). Extending a biologically inspired model of choice: Multi-alternatives, nonlinearity and value-based multidimensional choice. Philosophical Transactions of the Royal Society of London B, Series B: Biological Sciences, 362, 1655-1670.

Bousfield, W. A. (1953). The occurrence of clustering in the recall of randomly arranged associates. Journal of General Psychology, 49, 229-240.

Bower, G. H. (1972). Stimulus-sampling theory of encoding variability. In A. W. Melton \& E. Martin (Eds.), Coding processes in human memory (p. 85-121). New York: Wiley.

Brodie, D. A., \& Murdock, B. B. (1977). Effects of presentation time on nominal and functional serial position curves in free recall. Journal of Verbal Learning and Verbal Behavior, 16, 185-200.

Brown, G. D. A., Neath, I., \& Chater, N. (2007). A temporal ratio model of memory. Psychological Review, 114, 539-576.

Brown, G. D. A., Preece, T., \& Hulme, C. (2000). Oscillator-based memory for serial order. Psychological Review, 107, 127-181.

Brown, G. D. A., Sala, S., Foster, J., \& Vousden, J. (2007). Amnesia, rehearsal, and temporal distinctiveness models of recall. Psychonomic Bulletin \& Review, 14, 256-260.

Brown, T. (1824). Lectures on the philosophy of the human mind. Philadelphia: John Grigg and William P. Bason.

Burgess, N., \& Hitch, G. J. (1999). Memory for serial order: A network model of the phonological loop and its timing. Psychological Review, 106, 551-581.

Calkins, M. W. (1896). Association: An essay analytic and experimental. Psychological Review Monographs Supplement, 1(2), 1-56.

Carlesimo, G. A., Marfia, G. A., Loasses, A., \& Caltagirone, C. (1996) Recency effect in anterograde amnesia: Evidence for distinct memory stores underlying enhanced retrieval of terminal items in immediate and delayed recall paradigms. Neuropsychologia, 34, 177-184.

Chance, F. S., \& Abbott, L. F. (2000). Divisive inhibition in recurrent networks. Network, 11, 119-129.

Chance, F. S., Abbott, L. F., \& Reyes, A. D. (2002). Gain modulation from background synaptic input. Neuron, 35, 773-782.

Craik, F. I. M., \& Birtwistle, J. (1971). Proactive inhibition in free recall. Journal of Experimental Psychology, 91, 120-123.

Crowder, R. G. (1976). Principles of learning and memory. Hillsdale, NJ: Erlbaum.

Crowder, R. G. (1982). The demise of short-term memory. Acta Psychologica, 50, 291-323.

Davelaar, E. J., Goshen-Gottstein, Y., Ashkenazi, A., Haarmann, H. J., \& Usher, M. (2005). The demise of short-term memory revisited: Empirical and computational investigations of recency effects. Psychological Review, 112, 3-42.

Deese, J. (1959). On the prediction of occurrence of particular verbal intrusions in immediate recall. Journal of Experimental Psychology, 58, $17-22$.

Deese, J., \& Kaufman, R. A. (1957). Serial effects in recall of unorganized and sequentially organized verbal material. Journal of Experimental Psychology, 54, 180-187.

Egorov, A., Hamam, B., Fransen, E., Hasselmo, M., \& Alonso, A. A (2002, November 14). Graded persistent activity in entorhinal cortex neurons. Nature, 420, 173-178.

Eichenbaum, H., Otto, T., \& Cohen, N. J. (1994). Two functional components of the hippocampal memory system. Behavioral and Brain Sciences, 17, 449-518

Estes, W. K. (1950). Toward a statistical theory of learning. Psychological Review, 57, 94-107.

Estes, W. K. (1955). Statistical theory of spontaneous recovery and regression. Psychological Review, 62, 145-154. 
Estes, W. K. (1959). Component and pattern models with Markovian interpretations: Studies in mathematical learning theory. Stanford, CA: Stanford University Press.

Fransén, E., Tahvildari, B., Egorov, A. V., Hasselmo, M. E., \& Alonso, A. A. (2006). Mechanism of graded persistent cellular activity of entorhinal cortex layer V neurons. Neuron, 49, 735-746.

Glanzer, M., \& Cunitz, A. R. (1966). Two storage mechanisms in free recall. Journal of Verbal Learning and Verbal Behavior, 5, 351-360.

Glenberg, A. M., Bradley, M. M., Kraus, T. A., \& Renzaglia, G. J. (1983). Studies of the long-term recency effect: Support for a contextually guided retrieval theory. Journal of Experimental Psychology: Learning, Memory, and Cognition, 12, 413-418.

Glenberg, A. M., Bradley, M. M., Stevenson, J. A., Kraus, T. A., Tkachuk, M. J., \& Gretz, A. L. (1980). A two-process account of long-term serial position effects. Journal of Experimental Psychology: Human Learning and Memory, 6, 355-369.

Glenberg, A. M., \& Swanson, N. G. (1986). A temporal distinctiveness theory of recency and modality effects. Journal of Experimental Psychology: Learning, Memory, and Cognition, 12, 3-15.

Greene, R. L. (1986). Sources of recency effects in free recall. Psychological Bulletin, 99, 221-228.

Griffiths, T. L., Steyvers, M., \& Tenenbaum, J. B. (2007). Topics in semantic representation. Psycholgical Review, 114, 211-244.

Hasselmo, M. E., Bodelon, C., \& Wyble, B. P. (2002). A proposed function for hippocampal theta rhythm: Separate phases of encoding and retrieval enhance reversal of prior learning. Neural Computation, 14, 793-817.

Hogan, R. M. (1975). Interitem encoding and directed search in free recall. Memory \& Cognition, 3, 197-209.

Howard, M. W. (2004). Scaling behavior in the temporal context model. Journal of Mathematical Psychology, 48, 230-238.

Howard, M. W., Fotedar, M. S., Datey, A. V., \& Hasselmo, M. E. (2005). The temporal context model in spatial navigation and relational learning: Toward a common explanation of medial temporal lobe function across domains. Psychological Review, 112, 75-116.

Howard, M. W., \& Kahana, M. J. (1999). Contextual variability and serial position effects in free recall. Journal of Experimental Psychology: Learning, Memory, and Cognition, 25, 923-941.

Howard, M. W., \& Kahana, M. J. (2002a). A distributed representation of temporal context. Journal of Mathematical Psychology, 46, 269-299.

Howard, M. W., \& Kahana, M. J. (2002b). When does semantic similarity help episodic retrieval? Journal of Memory and Language, 46, 85-98.

Howard, M. W., Kahana, M. J., \& Wingfield, A. (2006). Aging and contextual binding: Modeling recency and lag-recency effects with the temporal context model. Psychonomic Bulletin \& Review, 13, 439-445.

Howard, M. W., Vankatadass, V., Norman, K. A., \& Kahana, M. J. (2007). Associative processes in immediate recency. Memory \& Cognition, 35, $1700-1711$

Howard, M. W., Youker, T. E., \& Venkatadass, V. (2008). The persistence of memory: Contiguity effects across several minutes. Psychonomic Bulletin \& Review, 15, 58-63.

Jones, M. N., \& Mewhort, D. J. K. (2007). Representing word meaning and order information in a composite holographic lexicon. Psychological Review, 114, 1-37.

Jonides, J., Lewis, R., Nee, D., Lustig, C., Berman, M., \& Moore, K. (2007). The mind and brain of short-term memory. Annual Review of Psychology, 59, 193-224.

Kahana, M. J. (1996). Associative retrieval processes in free recall. Memory \& Cognition, 24, 103-109.

Kahana, M. J. (in press). Foundations of human memory. Cambridge, England: Oxford University Press.

Kahana, M. J., Howard, M. W., \& Polyn, S. M. (2008). Associative retrieval processes in episodic memory. In H. L. Roediger III (Ed.), Cognitive psychology of memory. Vol. 2 of learning and memory: A comprehensive reference, 4 vols. Oxford, England: Elsevier.
Kahana, M. J., Howard, M. W., Zaromb, F., \& Wingfield, A. (2002). Age dissociates recency and lag recency effects in free recall. Journal of Experimental Psychology: Learning, Memory, and Cognition, 28, 530-540.

Kimball, D. R., Smith, T. A., \& Kahana, M. J. (2007). The fSAM model of false recall. Psychological Review, 114, 954-993.

Laming, D. (1999). Testing the idea of distinct storage mechanisms in memory. International Journal of Psychology, 34, 419-426.

Laming, D. (2006). Predicting free recalls. Journal of Experimental Psychology: Learning, Memory, and Cognition, 32, 1146-1163.

Landauer, T. K., \& Dumais, S. T. (1997). Solution to Plato's problem: The latent semantic analysis theory of acquisition, induction, and representation of knowledge. Psychological Review, 104, 211-240.

Lund, K., \& Burgess, C. (1996). Producing high-dimensional semantic spaces from lexical co-occurrence. Behavior Research Methods, Instruments, \& Computers, 28, 203-208.

Manns, J. R., Howard, M. W., \& Eichenbaum, H. (2007). Gradual changes in hippocampal activity support remembering the order of events. Neuron, 56, 530-540.

Marks, A. R., \& Cermak, L. S. (1998). Intact temporal memory in amnesic patients. Neuropsychologia, 36, 935-943.

McClelland, J. L., McNaughton, B. L., \& O’Reilly, R. C. (1995). Why there are complementary learning systems in the hippocampus and neocortex: Insights from the successes and failures of connectionist models of learning and memory. Psychological Review, 102, 419-457.

McGeoch, J. A. (1932). Forgetting and the law of disuse. Psychological Review, 39, 352-370.

McMillen, T., \& Holmes, P. (2006). The dynamics of choice among multiple alternatives. Journal of Mathematical Psychology, 50, 20-57.

Mensink, G-J. M., \& Raaijmakers, J. G. W. (1988). A model for interference and forgetting. Psychological Review, 95, 434-455.

Mensink, G-J. M., \& Raaijmakers, J. G. W. (1989). A model for contextual fluctuation. Journal of Mathematical Psychology, 33, 172-186.

Monsell, S. (1978). Recency, immediate recognition memory, and reaction time. Cognitive Psychology, 10, 465-501.

Murdock, B. B. (1960). The distinctiveness of stimuli. Psychological Review, 67, 16-31.

Murdock, B. B. (1962). The serial position effect of free recall. Journal of Experimental Psychology, 64, 482-488.

Murdock, B. B. (1974). Human memory: Theory and data. Potomac, MD: Erlbaum.

Murdock, B. B. (1997). Context and mediators in a theory of distributed associative memory (TODAM2). Psychological Review, 104, 839-862.

Murdock, B. B., \& Metcalfe, J. (1978). Controlled rehearsal in single-trial free recall. Journal of Verbal Learning and Verbal Behavior, 17, 309-324.

Murdock, B. B., \& Okada, R. (1970). Interresponse times in single-trial free recall. Journal of Verbal Learning and Verbal Behavior, 86, 263-267.

Nadel, L., \& Moscovitch, M. (1997). Memory consolidation, retrograde amnesia and the hippocampal complex. Current Opinion in Neurobiology, 7, 217-227.

Nadel, L., \& Moscovitch, M. (2001). The hippocampal complex and long-term memory revisited. Trends in Cognitive Sciences, 5, 228-230.

Nairne, J. S., Neath, I., Serra, M., \& Byun, E. (1997). Positional distinctiveness and the ratio rule in free recall. Journal of Memory and Language, 37, 155-166.

Neath, I. (1993). Contextual and distinctive processes and the serial position function. Journal of Memory and Language, 32, 820-840.

Neath, I., \& Crowder, R. G. (1990). Schedules of presentation and temporal distinctiveness in human memory. Journal of Experimental Psychology: Learning, Memory, and Cognition, 16, 316-327.

Neath, I., \& Crowder, R. G. (1996). Distinctiveness and very short-term serial position effects. Memory, 4, 225-242.

Nilsson, L. G., Wright, E., \& Murdock, B. B. (1975). The effects of visual presentation method on single-trial free recall. Memory \& Cognition, 3, 427-433. 
Paller, K. A., \& Wagner, A. D. (2002). Observing the transformation of experience into memory. Trends in Cognitive Sciences, 6, 93-102.

Postman, L., \& Phillips, L. W. (1965). Short-term temporal changes in free recall. Quarterly Journal of Experimental Psychology, 17, 132-138.

Raaijmakers, J. G. W. (1993). The story of the two-store model of memory: Past criticisms, current status, and future directions. In D. Meyer \& S. Kornblum (Eds.), Attention and performance xiv (p. 467-488). Cambridge, MA: MIT Press.

Raaijmakers, J. G. W., \& Shiffrin, R. M. (1980). SAM: A theory of probabilistic search of associative memory. In G. H. Bower (Ed.), The psychology of learning and motivation: Advances in research and theory (Vol. 14, p. 207-262). New York: Academic Press.

Raaijmakers, J. G. W., \& Shiffrin, R. M. (1981). Search of associative memory. Psychological Review, 88, 93-134.

Ratcliff, R. (1978). A theory of memory retrieval. Psychological Review, $85,59-108$.

Ratcliff, R. (2006). Modeling response signal and response time data. Cognitive Psychology, 53, 195-237.

Roediger, H. L., \& McDermott, K. B. (1995). Creating false memories: Remembering words not presented in lists. Journal of Experimental Psychology: Learning, Memory, and Cognition, 21, 803-814.

Romney, A. K., Brewer, D. D., \& Batchelder, W. H. (1993). Predicting clustering from semantic structure. Psychological Science, 4, 28-34.

Rundus, D. (1971). An analysis of rehearsal processes in free recall. Journal of Experimental Psychology, 89, 63-77.

Sederberg, P. B., Gauthier, L. V., Terushkin, V., Miller, J. F., Barnathan, J. A., \& Kahana, M. J. (2006). Oscillatory correlates of the primacy effect in episodic memory. NeuroImage, 32, 1422-1431.

Sederberg, P. B., Kahana, M. J., Howard, M. W., Donner, E. J., \& Madsen, J. R. (2003). Theta and gamma oscillations during encoding predict subsequent recall. Journal of Neuroscience, 23, 10809-10814.

Sederberg, P. B., Schulze-Bonhage, A., Madsen, J. R., Bromfield, E. B., Litt, B., Brandt, A., et al. (2007). Gamma oscillations distinguish true from false memories. Psychological Science, 18, 927-932.

Sederberg, P. B., Schulze-Bonhage, A., Madsen, J. R., Bromfield, E. B., McCarthy, D. C., Brandt, A., et al. (2007). Hippocampal and neocortical gamma oscillations predict memory formation in humans. Cerebral Cortex, 17, 1190-1196.

Shuell, T. J. (1969). Clustering and organization in free recall. Psychological Bulletin, 72, 353-374.

Siekmeier, P. J., Hasselmo, M. E., Howard, M. W., \& Coyle, J. (2007). Modeling of context-dependent retrieval in hippocampal region CA1:
Implications for cognitive function in schizophrenia. Schizophrenia Research, 89, 177-190.

Sirotin, Y. B., Kimball, D. R., \& Kahana, M. J. (2005). Going beyond a single list: Modeling the effects of prior experience on episodic free recall. Psychonomic Bulletin \& Review, 12, 787-805.

Smith, P. L., \& Ratcliff, R. (2004). Psychology and neurobiology of simple decisions. Trends in Neurosciences, 27, 161-168.

Squire, L. R. (1992). Memory and the hippocampus: A synthesis from findings with rats, monkeys, and humans. Psychological Review, 99, 195-231.

Tan, L., \& Ward, G. (2000). A recency-based account of the primacy effect in free recall. Journal of Experimental Psychology: Learning, Memory, and Cognition, 26, 1589-1626.

Thapar, A., \& Greene, R. L. (1993). Evidence against a short-term-store account of long-term recency effects. Memory \& Cognition, 21, 329-337.

Tzeng, O. J. L. (1973). Positive recency in delayed free recall. Journal of Verbal Learning and Verbal Behavior, 12, 436-439.

Underwood, B. J. (1983). Attributes of memory. Glenview, IL: Scott Foresman.

Usher, M., \& McClelland, J. L. (2001). The time course of perceptual choice: The leaky, competing accumulator model. Psychological Review, 108, 550-592.

van Vugt, M. K., Sekuler, R., Wilson, H. R., \& Kahana, M. J. (2008) Distinct electrophysiological correlates of proactive and similaritybased interference in visual working memory. Manuscript submitted for publication.

Ward, G., Woodward, G., Stevens, A., \& Stinson, C. (2003). Using overt rehearsals to explain word frequency effects in free recall. Journal of Experimental Psychology: Learning, Memory, and Cognition, 29, 186-210.

Watkins, M. J., Neath, I., \& Sechler, E. S. (1989). Recency effect in recall of a word list when an immediate memory task is performed after each word presentation. American Journal of Psychology, 102, 265-270.

Yntema, D. B., \& Trask, F. P. (1963). Recall as a search process. Journal of Verbal Learning and Verbal Behavior, 2, 65-74.

Zaromb, F. M., Howard, M. W., Dolan, E. D., Sirotin, Y. B., Tully, M., Wingfield, A., et al. (2006). Temporal associations and prior-list intrusions in free recall. Journal of Experimental Psychology: Learning, Memory, and Cognition, 32, 792-804.

Received August 14, 2007

Revision received February 29, 2008

Accepted March 1, 2008 